\title{
Lag, Anticipated, and Complete Synchronization and Cascade Control in the Dynamical Systems
}

\author{
Yin Li, ${ }^{1,2}$ Zheng Zeng, ${ }^{1}$ Yufeng Sun, ${ }^{1}$ and Ruiying Wei ${ }^{1}$ \\ ${ }^{1}$ School of Mathematics and Information Science, Shaoguan University, Shaoguan 512005, China \\ ${ }^{2}$ School of Mathematics and Computational Science, Sun Yat-sen University, Guangzhou 510275, China \\ Correspondence should be addressed to Yin Li; liyin2009521@163.com and Zheng Zeng; zz@sgu.edu.cn
}

Received 10 November 2013; Accepted 30 November 2013; Published 30 January 2014

Academic Editor: Ahmed El Wakil

Copyright (c) 2014 Yin Li et al. This is an open access article distributed under the Creative Commons Attribution License, which permits unrestricted use, distribution, and reproduction in any medium, provided the original work is properly cited.

\begin{abstract}
We obtain the lag, anticipated, and complete hybrid projective synchronization control (LACHPS) of dynamical systems to study the chaotic attractors and control problem of the chaotic systems. For illustration, we take the Colpitts oscillators as an example to achieve the analytical expressions of control functions. Numerical simulations are used to show the effectiveness of the proposed technique.
\end{abstract}

\section{Introduction}

Chaotic control and the structure of chaotic systems have attracted much attention in nonlinear sciences, especially in physics, chemistry, and biology. Different types of the structure and control method have been found in a variety of chaotic systems, such as constructing method [1-10], adaptive method [11], projective-Lag synchronization method [12-15], backstepping method [16], Q-S synchronization method [1720], and many others.

At the same time, many different types of synchronization in chaotic (hyperchaotic) systems were presented, for example, complete synchronization, generalized synchronization, phase synchronization, antisynchronization, general projective synchronization, lag synchronization, and anticipate synchronization, and so on.

To two dynamical systems, consider a full state hybrid projective synchronization (FSHPS) method [21], where the responses of the synchronized dynamical states synchronize up to a constant scaling matrix. In this paper, based on the Lyapunov stability theory, we propose a scheme of lag, anticipated, and complete hybrid projective synchronization control (LACHPS). In this method, every state variable of master system synchronizes other incompatible state variables of slave system; particularly, for oscillators, two different designs are shown.
When $x(t)$ and $y(t)$ are the state vectors of two $n$ dimensional chaotic systems. These two systems are completely synchronized [22] if the synchronization error $\| y(t)-$ $x(t) \| \rightarrow 0$ as $t \rightarrow \infty$. AS [23] is defined when the error $\|y(t)+x(t)\| \rightarrow 0$ as $t \rightarrow \infty$. PS [24] is a situation in which the state vectors $x(t)$ and $y(t)$ synchronize up to a constant factor $\alpha$ (i.e. $\|y(t)-\alpha x(t)\| \rightarrow 0$ as $t \rightarrow \infty$. MPS [25] is defined if the state vectors of two systems synchronize up to a constant scaling matrix which means that $\|y(t)-M x(t)\| \rightarrow$ 0 as $t \rightarrow \infty$. LS [13] implies that the state variables of the two coupled chaotic systems become synchronized but with a time lag with respect to each other; that is, $\| y(t)-x(t-$ $\tau) \| \rightarrow 0$ as $t \rightarrow \infty$, where $\tau$ is the positive time lag. PLS has been introduced recently in $[15,26-28]$ as $\| y(t)-\beta x(t-$ $\tau) \| \rightarrow 0$ as $t \rightarrow \infty$, where $\beta$ is a constant scaling factor. Synchronization can be addressed as a stabilization problem. This means that the trajectories of the synchronization error have to be stabilized at the origin.

In realistic and engineering applications, LS and PLS always affect the dynamical behaviors of chaotic systems. For example, in the telephone communication system, the voice one hears on the receiver side at time $t$ is the voice from the transmitter side at time $t$. LS and PLS have been recently studied on systems described in [15, 28-30]. For more details about chaotic control see [31-38] and for the elements of the cyclicity theory of planar systems see [39-41]. Our goal in 
TABLE 1

\begin{tabular}{lcc}
\hline Setting the matrix $M$ & Setting the matrix $N$ and the value of $\tau$ & Type of synchronization \\
\hline$M=\operatorname{diag}\left(\delta_{1}, \delta_{2}, \ldots, \delta_{n}\right)$ & $N=\operatorname{diag}\left(\epsilon_{1}, \epsilon_{2}, \ldots, \epsilon_{n}\right), \tau=0$ & HPS \\
$M=\operatorname{diag}(1,1, \ldots, 1)$ & $N=\operatorname{diag}\left(\epsilon_{1}, \epsilon_{2}, \ldots, \epsilon_{n}\right), \tau>0$ & PLS \\
$M=\operatorname{diag}(1,1, \ldots, 1)$ & $N=\operatorname{diag}\left(\epsilon_{1}, \epsilon_{2}, \ldots, \epsilon_{n}\right), \tau=0$ & PS \\
$M=\operatorname{diag}(1,1, \ldots, 1)$ & $N=\operatorname{diag}(1,1,1, \ldots, 1), \tau>0$ & LS \\
$M=\operatorname{diag}(1,1, \ldots, 1)$ & $N=\operatorname{diag}(1,1,1, \ldots, 1), \tau=0$ & CS \\
$M=\operatorname{diag}(1,1, \ldots, 1)$ & $N=\operatorname{diag}\left(f_{1}, f_{2}, \ldots, f_{n}\right), \tau=0$ & FS \\
\hline
\end{tabular}

this paper is to introduce and investigate the lag, anticipated, and complete hybrid projective synchronization control (LACHPS) of two $n$-dimensional nonlinear systems.

Definition 1. For $n$-dimensional master and slave nonlinear systems as $\dot{x}=F(x, t), \dot{y}=G(y, t)+U$, where $x=$ $\left(x_{1}(t), x_{2}(t), \ldots, x_{n}(t)\right)^{T}, y=\left(y_{1}(t), y_{2}(t), \ldots, y_{n}(t)\right)^{T}, U=$ $\left(u_{1}(x, y), u_{2}(x, y), \ldots, u_{n}(x, y)\right)$ is a controller to be determined later. The LACHPS is defined if the synchronization error $\lim _{t \rightarrow \infty}\|M y(t)-N x(t-\tau)\| \rightarrow 0$, where $x(t)$ and $y(t)$ are the state vectors of two systems, the matrixes $M$ and $N$ are defined as $M=\operatorname{diag}\left(\delta_{1}, \delta_{2}, \ldots, \delta_{n}\right)$ and $N=\operatorname{diag}\left(\epsilon_{1}, \epsilon_{2}, \ldots, \epsilon_{n}\right), \delta_{i}\left(\epsilon_{i}\right)=$ constants or a "scaling function matrix" ( $i=1,2, \ldots, n)$, and $\tau$ is the time lag or anticipated. It is said that the master system and slave system are globally (i) lag hybrid projective synchronization control ( $\tau>0, \tau$ is called the synchronization lag); (ii) hybrid complete projective synchronization control ( $\tau=0)$; and (iii) anticipated hybrid projective synchronization control $(\tau<$ $0,-\tau>0$ is called the synchronization anticipation).

We remark that the above-mentioned types of synchronization are special cases of our definition. Table 1 illustrates these types of synchronization.

In order to show the results of LACHPS of two nonlinear systems, we choose the chaotic Colpitts oscillators as an example.

This paper is organized as follows. In Section 2, we show the general scheme description and theorem. In Sections 3 and 4, the Colpitts oscillator as a example is shown via applications of the LACHPS control method and cascade method. And numerical simulations are used to show the effectiveness. Finally, conclusions are drawn.

\section{The Extended Control Method and the Main Results}

In this section, the extended hybrid projective control method is designed to achieve synchronization control based on [42-49] method. Consider the master system in the form of

$$
\dot{x}(t)=\Phi x(t)+F(x(t), t),
$$

where $x(t) \in R^{n}, \Phi$ is an $m \times m$ constant matrix, and $F$ : $R^{n} \rightarrow R^{n}$ is a nonlinear function. Assume that the slave system coupled with (1) is as follows:

$$
\dot{y}(t)=\Phi y(t)+G(y(t), t)+U,
$$

where $y(t) \in R^{n}$, and $U$ is a controller to be determined later. Denote $e_{i}=\delta_{i} x_{i}(t)-\epsilon_{i} y_{i}(t-\tau)(i=1,2, \ldots, n)$ and $\delta_{i}\left(\epsilon_{i}\right)=$ constants or a scaling function matrix. If $\lim _{t \rightarrow \infty}\|e\|=0, e=$ $\left(e_{1}, e_{2}, \ldots, e_{n}\right)$, these two chaotic systems can be controlled via the LACHPS.

Proposition 2. When the matrices $M=\operatorname{diag}\left(\delta_{1}, \delta_{2}, \ldots, \delta_{n}\right)$ and $N=\operatorname{diag}\left(\epsilon_{1}, \epsilon_{2}, \ldots, \epsilon_{n}\right)$ are two invertible diagonal function matrices; lag, anticipated, and complete hybrid projective synchronization between the two systems (1) and (2) will occur, if the following conditions are satisfied:

(i)

$$
\begin{aligned}
U= & N^{-1}(g x(t)-h y(t-\tau))+N^{-1} M F(x(t), t) \\
& -G(y(t-\tau), t)+N^{-1} K[M x(t)-N y(t-\tau)], \\
& \text { where } g=\operatorname{diag}\left(\dot{\delta}_{1}, \dot{\delta}_{2}, \ldots, \dot{\delta}_{n}\right), h=\operatorname{diag}\left(\dot{\epsilon}_{1}, \dot{\epsilon}_{2}, \ldots, \dot{\epsilon}_{n}\right) \\
& \text { and } K \in R^{n \times n} ; \\
\text { (ii) the real parts of all the eigenvalues of }(\Phi+K) \text { are } & \text { negative. }
\end{aligned}
$$

Proof. According to $e=M x(t)-N y(t-\tau)$ in definition of LACHPS, one can get

$$
\begin{aligned}
\dot{e}(t)= & \dot{M} x(t)+M \dot{x}(t)-\dot{N} y(t-\tau)-N \dot{y}(t-\tau) \\
= & g x(t)+M[\Phi x(t)+F(x(t), t)]-h y(t-\tau) \\
& -N(\Phi y(t-\tau)+G(y(t-\tau), t)+U) \\
= & \Phi(M x(t)-N y(t-\tau))+g x(t)+M F(x(t), t) \\
& -h y(t-\tau)-N G(y(t-\tau), t) \\
& -N\left[N^{-1}(g x(t)-h y(t-\tau))+N^{-1} M F(x(t), t)\right. \\
& \quad-G(y(t-\tau), t) \\
& \left.+N^{-1} K(M x(t)-N y(t-\tau))\right] \\
= & (\Phi+K) e(t) .
\end{aligned}
$$

We solve the above equation $\dot{e}(t)=(\Phi+K) e(t)$, and

$$
\|e(t)\|=\left\|e^{(\Phi+K) t} e(0)\right\| .
$$

Because the real parts of all the eigenvalues of $(\Phi+K)$ are negative, $\|e(t)\| \rightarrow 0$ if $t \rightarrow 0$. Namely, $\lim _{t \rightarrow 0}\|e(t)\|=0$. 
For a feasible control, the feedback $K$ must be selected such that all the eigenvalues of $(\Phi+K)$, if any, have negative real parts. Thus, if the matrix $(\Phi+K)$ is in full rank, the system $\dot{e}$ is asymptotically stable at the origin, which implies that (1) and (2) are in the state of LACHPS control.

Proposition 3. Let a constant matrix $M$ and a diagonal function matrix $N=\operatorname{diag}\left(\epsilon_{1}, \epsilon_{2}, \ldots, \epsilon_{n}\right)$; lag, anticipated, and complete hybrid projective synchronization between the two systems (1) and (2) will occur, if the following conditions are satisfied:

(i)

$$
\begin{aligned}
U= & -N^{-1} h y(t-\tau)+N^{-1} M F(x(t), t) \\
& -G(y(t-\tau), t)-N^{-1} K[M x(t)-N y(t-\tau)],
\end{aligned}
$$

where $M=\operatorname{diag}\left(\delta_{1}, \delta_{2}, \ldots, \delta_{n}\right), h=\operatorname{diag}\left(\dot{\epsilon}_{1}, \dot{\epsilon}_{2}, \ldots\right.$, $\left.\dot{\epsilon}_{n}\right)$, and $K \in R^{n \times n}$;

(ii) the real parts of all the eigenvalues of $(\Phi+K)$ are negative.

Similar to the way of Theorem 5, the proof of Proposition 3 is straightforward in Appendix.

Proposition 4. Let a diagonal function matrix $M=\operatorname{diag}\left(\delta_{1}\right.$, $\left.\delta_{2}, \ldots, \delta_{n}\right)$ and a constant matrix $N$; the extended hybrid projective synchronization control between the two systems (1) and (2) will occur, if the following conditions are satisfied:

(i)

$$
\begin{aligned}
U= & -N^{-1} g x(t)+N^{-1} M F(x(t), t) \\
& -G(y(t-\tau), t)-N^{-1} K[M x(t)-N y(t-\tau)],
\end{aligned}
$$

where $g=\operatorname{diag}\left(\dot{\delta}_{1}, \dot{\delta}_{2}, \ldots, \dot{\delta}_{n}\right), h=\operatorname{diag}\left(\epsilon_{1}, \epsilon_{2}, \ldots, \epsilon_{n}\right)$, and $K \in R^{n \times n}$;

(ii) The real parts of all the eigenvalues of $(\Phi+K)$ are negative.

Similar to the way of the Proposition 2, the proof of Proposition 4 is straightforward in Appendix.

In order to choose a suitable control law $U$ or a vector function $K$, and $\dot{e}(t)=(\Phi+K) e(t)$ is asymptotically stable, we give the following theorem such that systems (1) and (2) are in the state of LACHPS control.

Theorem 5. If the conditions are satisfied $P(\Phi+K)+(\Phi+$ $K)^{H} P=-Q$, lag, anticipated, and complete hybrid projective synchronization between the two systems (1) and (2) can be achieved, where $P, Q$ are real symmetric positive definite matrix, $K \in R^{n \times n}, H$ stands for conjugate transpose of a matrix.

Proof. According to $e=M x(t)-N y(t-\tau)$ in definition of LACHPS, one can get

$$
\dot{e}(t)=(\Phi+K) e(t) .
$$

If $\lambda$ is one of the eigenvalues of matrix $\Phi+K$ and the corresponding nonzero eigenvector is $\beta$,

$$
(\Phi+K) \beta=\lambda \beta \text {. }
$$

Multiplying the above equation left by $\beta^{H} P$, we obtain

$$
\beta^{H} P(\Phi+K) \beta=\beta^{H} P \lambda \beta \text {. }
$$

Similarly, we also can derive that

$$
\left[\beta^{H}(\Phi+K)^{H}\right] P \beta=\bar{\lambda} \beta^{H} P \beta .
$$

From the above two equations, we can obtain

$$
\lambda+\bar{\lambda}=\frac{\beta^{H}\left[P(\Phi+K)+(\Phi+K)^{H} P\right] \beta}{\beta^{H} P \beta} .
$$

Since $\beta^{H}\left[P(\Phi+K)+(\Phi+K)^{H} P\right] \beta=-Q$, and $P$ and $Q$ are real symmetric positive definite matrix,

$$
\begin{gathered}
\beta^{H} P \beta>0, \quad \beta^{H} Q \beta>0, \\
\lambda+\bar{\lambda}=-\frac{\beta^{H} Q \beta}{\beta^{H} P \beta}<0 .
\end{gathered}
$$

According to the stability theory, the system $\dot{e}$ is asymptotically stable at the origin.

Remark 6. If we rewrite (2) as

$$
\dot{y}(t)=\theta y(t)+G(y(t), t)+U
$$

we can obtain the following results.

(1) Let two invertible diagonal function matrix $M=$ $\operatorname{diag}\left(\delta_{1}, \delta_{2}, \ldots, \delta_{n}\right)$ and $N=\operatorname{diag}\left(\epsilon_{1}, \epsilon_{2}, \ldots, \epsilon_{n}\right) ;$ lag, anticipated, and complete hybrid projective synchronization between the two systems (1) and (2) will occur, if the following conditions are satisfied:

(i)

$$
\begin{aligned}
U= & N^{-1}(g x(t)-h y(t-\tau))+N^{-1} M F(x(t), t) \\
& -G(y(t-\tau), t)+(\phi+K) y(t-\tau) \\
& -N^{-1}(\theta+K) M x(t),
\end{aligned}
$$

where $g=\operatorname{diag}\left(\dot{\delta}_{1}, \dot{\delta}_{2}, \ldots, \dot{\delta}_{n}\right), h=\operatorname{diag}\left(\dot{\epsilon}_{1}\right.$, $\left.\dot{\epsilon}_{2}, \ldots, \dot{\epsilon}_{n}\right), \theta \in R^{n \times n}$, and $K \in R^{n \times n}$.

(2) Let a constant matrix $M$ and a diagonal function matrix $N=\operatorname{diag}\left(\epsilon_{1}, \epsilon_{2}, \ldots, \epsilon_{n}\right)$; lag, anticipated, and complete hybrid projective synchronization between the two systems (1) and (2) will occur, if the following conditions are satisfied:

(ii)

$$
\begin{aligned}
U= & N^{-1}[M F(x(t), t)-h y(t-\tau)]-G(y(t-\tau), t) \\
& +N^{-1}(\theta+K)[M x(t)-N y(t-\tau)],
\end{aligned}
$$

where $M=\operatorname{diag}\left(\delta_{1}, \delta_{2}, \ldots, \delta_{n}\right), h=\operatorname{diag}\left(\dot{\epsilon}_{1}\right.$, $\left.\dot{\epsilon}_{2}, \ldots, \dot{\epsilon}_{n}\right), \theta \in R^{n \times n}$, and $K \in R^{n \times n}$.

(3) Let a diagonal function matrix $M=\operatorname{diag}\left(\delta_{1}, \delta_{2}, \ldots\right.$, $\delta_{n}$ ) and a constant matrix $N$; lag, anticipated, and complete hybrid projective synchronization between the two systems (1) and (2) will occur, if the following conditions are satisfied: 
(iii)

$$
\begin{aligned}
U= & N^{-1} M F(x(t), t)+N^{-1} g x(t)-G(y(t-\tau), t) \\
& -N^{-1}(\theta+K) M x(t)+(\theta+K) y(t-\tau)
\end{aligned}
$$

where $g=\operatorname{diag}\left(\dot{\delta}_{1}, \dot{\delta}_{2}, \ldots, \dot{\delta}_{n}\right), \theta \in R^{n \times n}$, and $K \in R^{n \times n}$.

(iv) The real parts of all the eigenvalues of $(\phi+\theta+K)$ are negative with Cases 1-3.

\section{Applications of the LACHPS Control Method}

Now, we introduce the following nonlinear system:

$$
\begin{gathered}
\dot{x}(t)=a z(t)-c|y(t)|+d, \\
\dot{y}(t)=a z(t), \\
\dot{z}(t)=-\frac{x(t)}{2 a}-\frac{y(t)}{2 a}-b z(t),
\end{gathered}
$$

where $a, b, c$, and $d$ are real constants, if $a=-2, b=0.4$, $c=1.62$, and $d=3$, the simulation results of system (18) with the initial conditions $(0,0,0)$. System (18) has chaotic attractor as shown in Figures 1 and 2. System (18) temporal evolution of the state variables is shown in Figure 3. For more detailed dynamical properties of system (2), the reader should refer to [50].

In the following, we rewrite the chaotic system (18) as a master system:

$$
\begin{gathered}
\dot{x}_{m}(t)=a z_{m}(t)-c\left|y_{m}(t)\right|+d, \\
\dot{y}_{m}(t)=a z_{m}(t), \\
\dot{z}_{m}(t)=-\frac{x_{m}(t)}{2 a}-\frac{y_{m}(t)}{2 a}-b z_{m}(t)
\end{gathered}
$$

and the system related to (20), given by

$$
\begin{gathered}
\dot{x}_{s}(t)=a z_{s}(t)-c\left|y_{s}(t)\right|+d+u_{1}, \\
\dot{y}_{s}(t)=a z_{s}(t)+u_{2}, \\
\dot{z}_{s}(t)=-\frac{x_{s}(t)}{2 a}-\frac{y_{s}(t)}{2 a}-b z_{s}(t)+u_{3}
\end{gathered}
$$

as a slave system, where the subscripts " $m$ " and " $s$ " stand for the master system and slave system, respectively. Let the error state be

$$
\begin{aligned}
e(t)= & \left(e_{1}, e_{2}, e_{3}\right)^{T} \\
= & {\left[\delta_{1} x_{m}(t)-\epsilon_{1} x_{s}(t-\tau),\right.} \\
& \delta_{2} y_{m}(t)-\epsilon_{2} y_{s}(t-\tau), \\
& \left.\delta_{3} z_{m}(t)-\epsilon_{3} z_{s}(t-\tau)\right]^{T} .
\end{aligned}
$$

Then from (18) and (19), we obtain the error system

$$
\begin{aligned}
\dot{e}_{1}= & \delta_{1}\left[a z_{m}(t)-c\left|y_{m}(t)\right|+d\right] \\
& -\epsilon_{1}\left[a z_{s}(t-\tau)-c\left|y_{s}(t-\tau)\right|+d+u_{1}\right],
\end{aligned}
$$

$$
\begin{gathered}
\dot{e}_{2}=\delta_{2} a z_{m}(t)-\epsilon_{2} a z_{s}(t-\tau)-\epsilon_{2} u_{2}, \\
\dot{e}_{3}=\delta_{3}\left[-\frac{x_{m}(t)}{2 a}-\frac{y_{m}(t)}{2 a}-b z_{m}(t)\right] \\
-\epsilon_{3}\left[-\frac{x_{s}(t-\tau)}{2 a}-\frac{y_{s}(t-\tau)}{2 a}-b z_{s}(t-\tau)+u_{3}\right] .
\end{gathered}
$$

To the LACHPS synchronization control between systems (18) and (19), we have the following theorem.

Proposition 7. For the chaotic Colpitts oscillator (18), if one of the following feedback controllers $u_{i}(i=1,2,3)$ is chosen for the slave system (19)

$$
\begin{aligned}
& u_{1}=\left(\delta_{1} a z_{m}(t)-\delta_{1} c\left|y_{m}(t)\right|+\delta_{1} d-\epsilon_{1} a z_{s}(t-\tau)\right. \\
& \left.+\epsilon_{1} c\left|y_{s}(t-\tau)\right|-\epsilon_{1} d-a e_{3}\right) \times \epsilon_{1}^{-1}, \\
& u_{2}=\frac{\delta_{2} a z_{m}(t)-\epsilon_{2} a z_{s}(t-\tau)-a e_{3}+e_{2}}{\epsilon_{2}}, \\
& u_{3}=\left(-\delta_{3} x_{m}(t)-\delta_{3} y_{m}(t)-2 \delta_{3} b z_{m}(t) a\right. \\
& \left.+\epsilon_{3} x_{s}(t-\tau)+\epsilon_{3} y_{s}(t-\tau)\right) \times\left(2 \epsilon_{3} a\right)^{-1} \\
& +\frac{2 \epsilon_{3} b z_{s}(t-\tau) a+e_{1}+e_{2}+2 b e_{3} a}{2 \epsilon_{3} a}, \\
& u_{1}=\frac{\left(\epsilon_{3} a-\epsilon_{1} a\right) z_{s}(t-\tau)}{\epsilon_{1}}+\frac{\left(\epsilon_{1} c-c \epsilon_{2}\right)\left|y_{s}(t-\tau)\right|}{\epsilon_{1}} \\
& +\frac{\left(\delta_{1} a-a \delta_{3}\right) z_{m}(t)}{\epsilon_{1}} \\
& +\frac{\left(c \delta_{2}-\delta_{1} c\right)\left|y_{m}(t)\right|}{\epsilon_{1}}+\frac{-\epsilon_{1} d+\delta_{1} d}{\epsilon_{1}} \\
& u_{2}=\frac{\left(-\epsilon_{2} a+a \epsilon_{3}\right) z_{s}(t-\tau)}{\epsilon_{2}}+\frac{\left(-a \delta_{3}+\delta_{2} a\right) z_{m}(t)}{\epsilon_{2}} \\
& +\left(-\delta_{1} x_{m}(t)-\delta_{2}\left|y_{m}(t)\right|\right. \\
& \left.+\epsilon_{2}\left|y_{s}(t-\tau)\right|+\epsilon_{1} x_{s}(t-\tau)\right) \times \epsilon_{2}^{-1}, \\
& u_{3}=\frac{\left(-2 \epsilon_{3} a+2 \epsilon_{3} b a\right) z_{s}(t-\tau)}{2 \epsilon_{3} a} \\
& +\frac{\left(\epsilon_{3}-2 \epsilon_{2} a\right)\left|y_{s}(t-\tau)\right|}{2 \epsilon_{3} a}+\frac{\left(\epsilon_{3}-2 \epsilon_{1} a\right) x_{s}(t-\tau)}{2 \epsilon_{3} a} \\
& +\frac{\left(-2 \delta_{3} b a+2 \delta_{3} a\right) z_{m}(t)}{2 \epsilon_{3} a}+\frac{\left(-\delta_{3}+2 \delta_{2} a\right)\left|y_{m}(t)\right|}{2 \epsilon_{3} a} \\
& +\frac{\left(2 \delta_{1} a-\delta_{3}\right) x_{m}(t)}{2 \epsilon_{3} a},
\end{aligned}
$$

where $a\langle 0, b\rangle 0, c\rangle 0, d\rangle 0$, and $\epsilon_{i}$ and $\delta_{i}$ are real, then the zero solution of the error system (21) is globally stable, and 


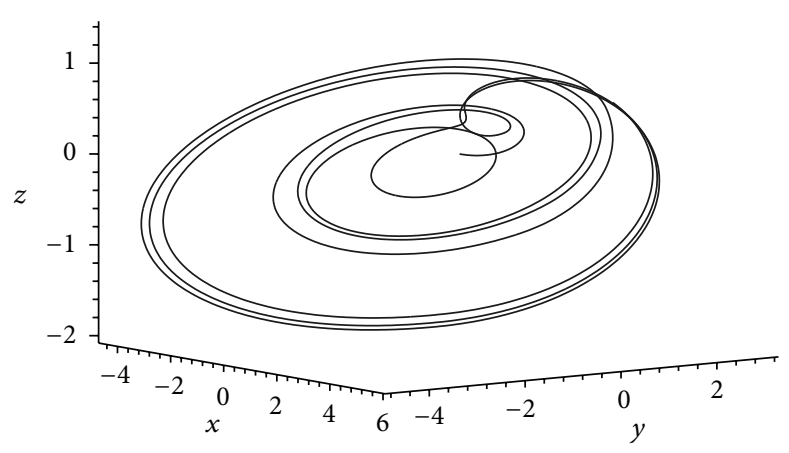

(a)

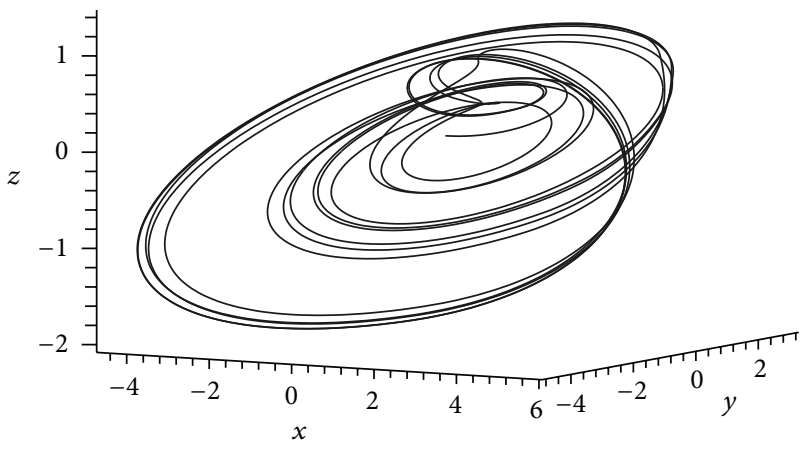

(b)

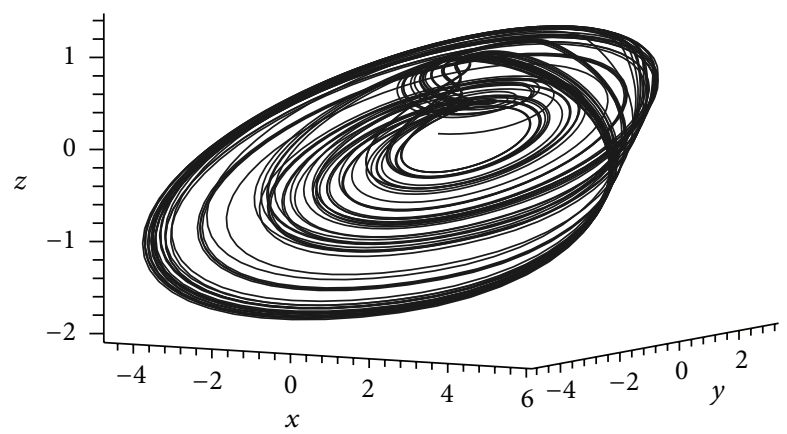

(c)

FIgURE 1: Chaotic attractors for the Colpitts system with temporal evolution in different 3D spaces.

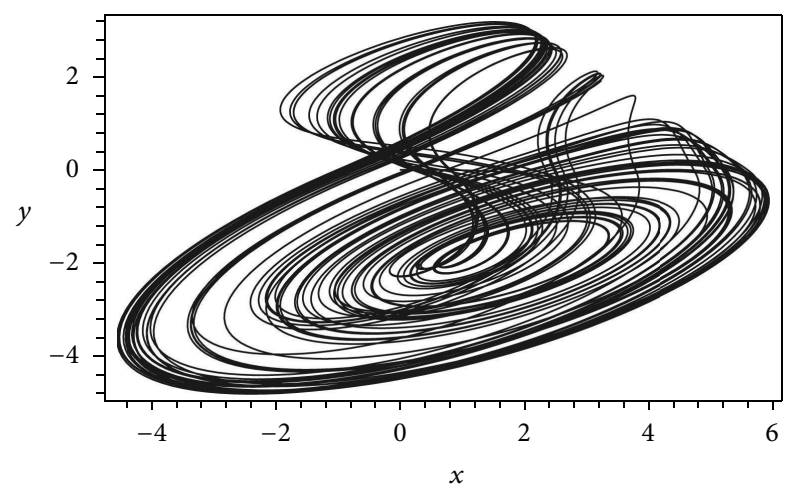

(a)

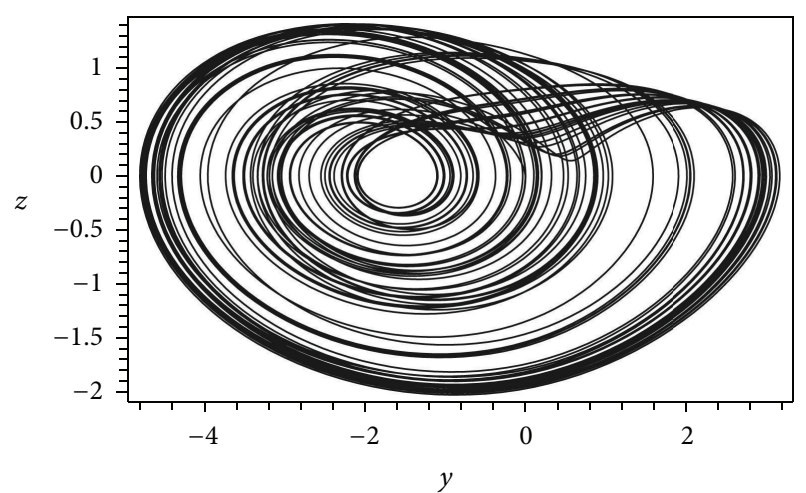

(b)

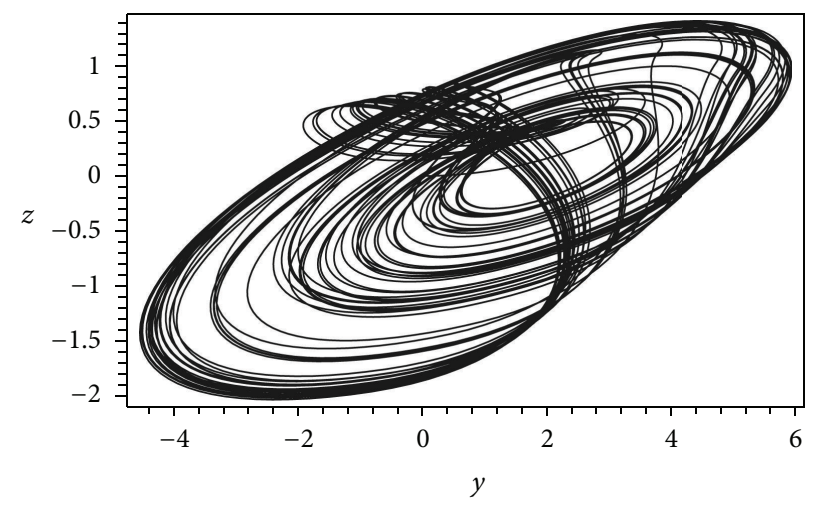

(c)

FIGURE 2: The phase figure for the Colpitts system with temporal evolution in different plane. 


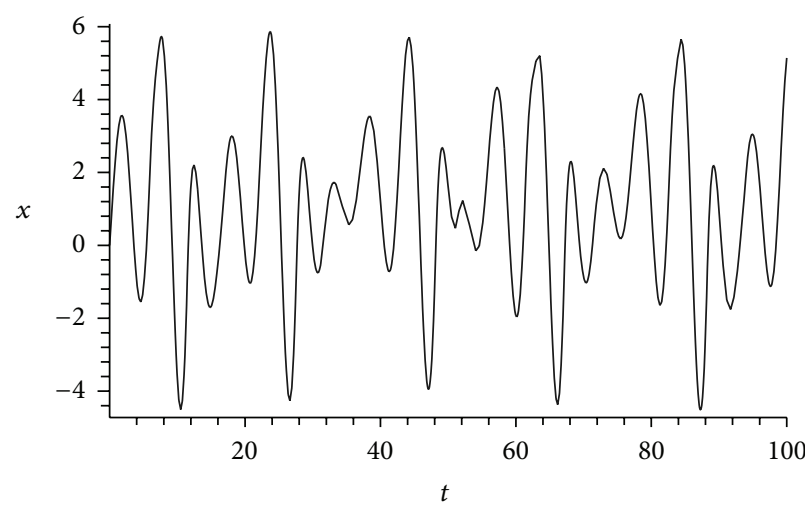

(a)

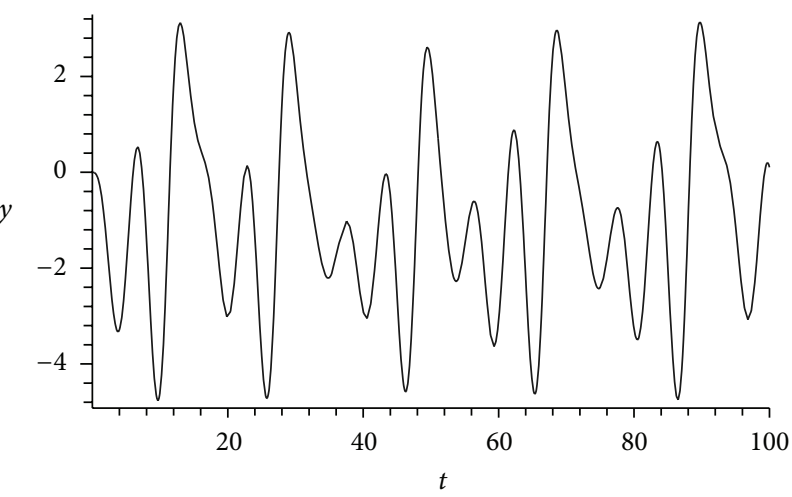

(b)

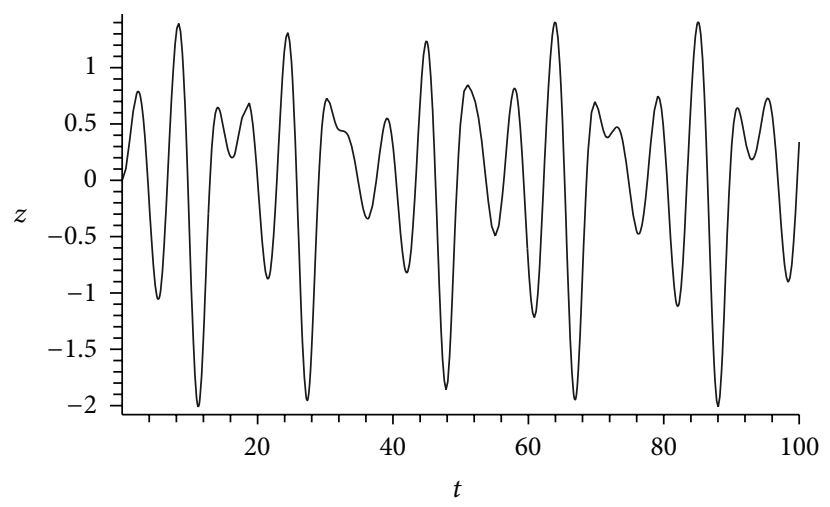

(c)

FIgURE 3: The temporal evolution of the state variables.

thus (i) globally lag synchronization for $\tau<0$, (ii) anticipated synchronization for $\tau>0$, and (iii) complete synchronization for $\tau=0$ occur between the master system (18) and the slave system (19).

Proof. Similar to the way of Propositions 2-4, the proof of Proposition 7 is straightforward and we omit the detail steps. We give another proof method via Lyapunov function in the following.

Consider the controller (22) and choose the following quadratic form, positive definite of Lyapunov function:

$$
V\left(e_{1}, e_{2}, e_{3}\right)=\frac{1}{2}\left(e_{1}^{2}+e_{2}^{2}+e_{3}^{2}\right)
$$

which implies that $P=\operatorname{diag}(1 / 2,1 / 2,1 / 2)$ and thus $\lambda_{\min }(P)=$ $1 / 2, \lambda_{\max }(P)=1 / 2$. Differentiating $V(t)$ along the trajectory of system (21) yields

$$
\begin{aligned}
\left.\frac{d V(t)}{d t}\right|_{(21)}= & e_{1} \dot{e}_{1}+e_{2} \dot{e}_{2}+e_{3} \dot{e}_{3} \\
= & e_{1}\left[\delta_{1}\left(a z_{m}(t)-c\left|y_{m}(t)\right|+d\right)\right. \\
& \left.\quad-\epsilon_{1}\left(a z_{s}(t-\tau)-c\left|y_{s}(t-\tau)\right|+d+u_{1}\right)\right] \\
& +e_{2}\left[\delta_{2} a z_{m}(t)-\epsilon_{2} a z_{s}(t-\tau)-u_{2}\right] \\
& +e_{3}\left[\delta_{3}\left(-\frac{x_{m}(t)}{2 a}-\frac{y_{m}(t)}{2 a}-b z_{m}(t)\right)\right]
\end{aligned}
$$

$$
\begin{gathered}
-e_{3}\left[\epsilon _ { 3 } \left(-\frac{x_{s}(t-\tau)}{2 a}-\frac{y_{s}(t-\tau)}{2 a}\right.\right. \\
\left.\left.-b z_{s}(t-\tau)+u_{3}\right)\right] .
\end{gathered}
$$

We put $u_{1}, u_{2}$, and $u_{3}$ into (25) and then simplify and yield

$$
\begin{aligned}
\left.\frac{d V(t)}{d t}\right|_{(21)}= & a e_{1} e_{3}+e_{2}\left(a e_{3}-e_{2}\right) \\
& +e_{3}\left(\frac{-e_{1}}{2 a}-\frac{-1}{2 a} e_{2}-b e_{3}\right) \\
= & -e_{2}^{2}-b e_{3}^{2}+\left(a-\frac{1}{2 a}\right) e_{1} e_{3}+\left(a-\frac{1}{2 a}\right) e_{2} e_{3} \\
\leq & \left(\frac{a}{2}-\frac{1}{4 a}\right) e_{1}^{2}+\left(\frac{a}{2}-\frac{1}{4 a}-1\right) e_{2}^{2} \\
& +\left(a-\frac{1}{2 a}-b\right) e_{3}^{2},
\end{aligned}
$$

where $Q=\operatorname{diag}(a / 2-1 / 4 a, a / 2-1 / 4 a-1, a-1 / 2 a-b)$. Then using Lemma 1 [20], we have the estimation

$$
\begin{aligned}
e_{1}^{2}+e_{2}^{2} & +e_{3}^{2} \\
& \leq \frac{\lambda_{\max }}{\lambda_{\min }}\left[e_{1}^{2}(0)+e_{2}^{2}(0)+e_{3}^{2}(0)\right] e^{-\left(\lambda_{\min }(\mathrm{Q}) / \lambda_{\max }(P)\right)\left(t-t_{0}\right)}
\end{aligned}
$$




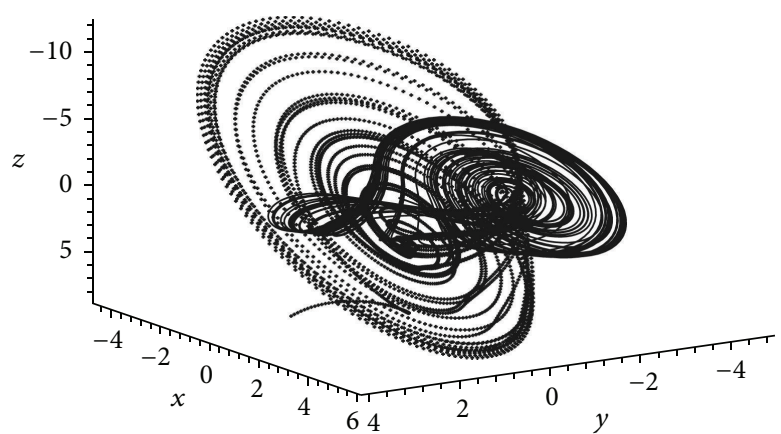

(a)

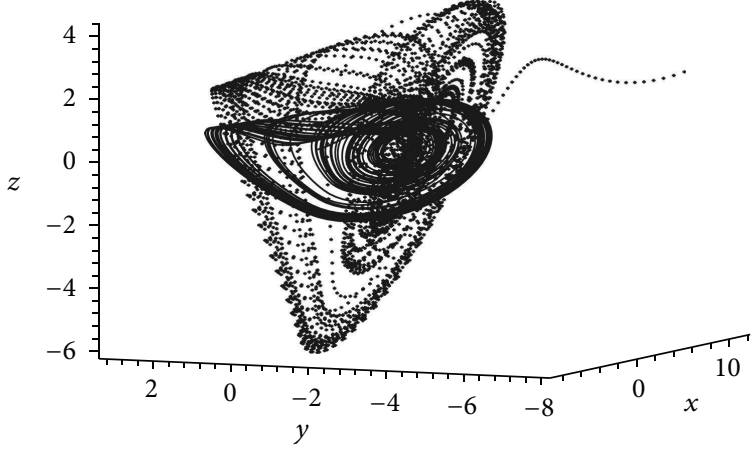

(b)

Figure 4: (a) The synchronized attractors in $(x, y, z)$ space and (b) the synchronized attractors with scaling factor in $(x, y, z)$ space, “ - -" denotes for the master system, “..." denotes for the slave system synchronized.

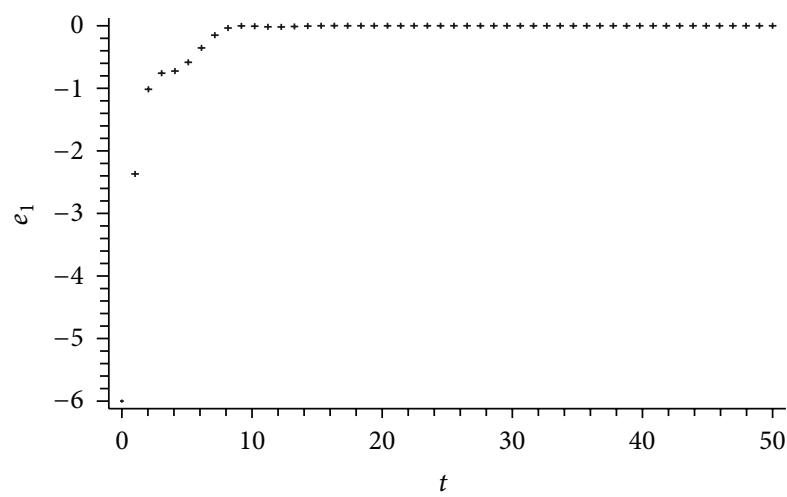

(a)

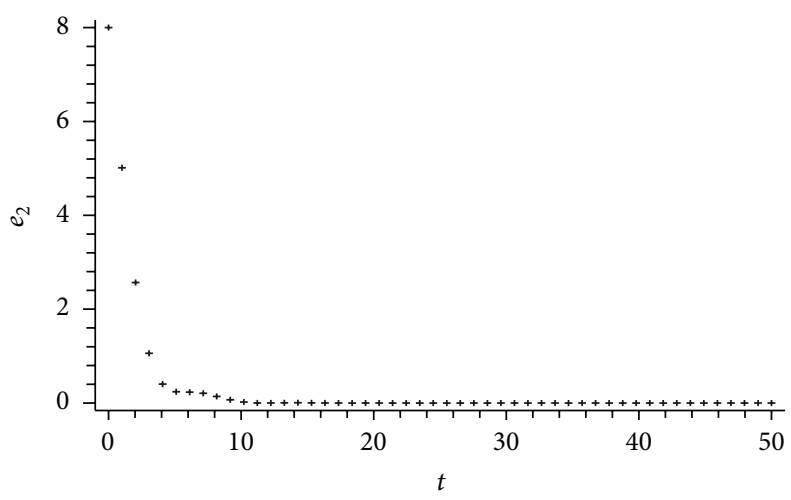

(b)

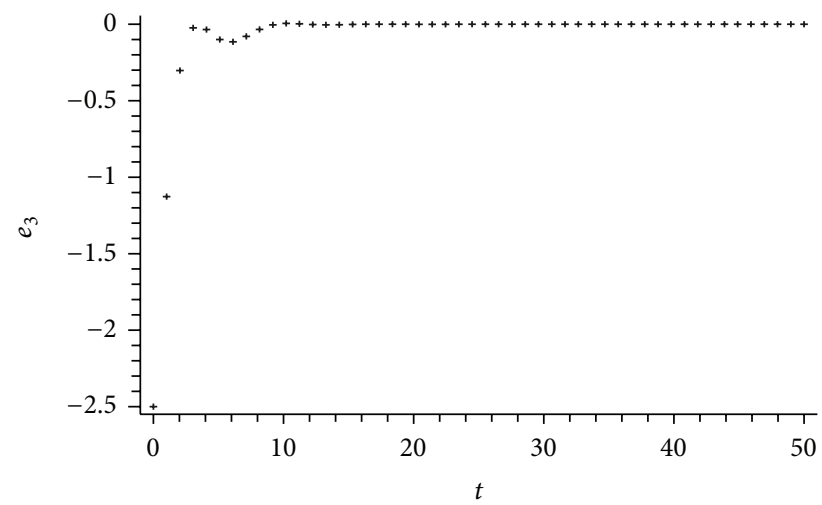

(c)

FIgURE 5: The orbits of error states: (a) $e_{1}=\delta_{1} x_{m}(t)-\epsilon_{1} x_{s}(t-\tau)$, (b) $e_{2}=\delta_{2} y_{m}(t)-\epsilon_{2} y_{s}(t-\tau)$, and (c) $e_{3}=\delta_{3} z_{m}(t)-\epsilon_{3} z_{s}(t-\tau)$.

$$
\begin{aligned}
= & {\left[e_{1}^{2}(0)+e_{2}^{2}(0)+e_{3}^{2}(0)\right] } \\
& \times e^{-2\left(\lambda_{\min }(a / 2-1 / 4 a, a / 2-1 / 4 a-1, a-1 / 2 a-b)\right)\left(t-t_{0}\right)} .
\end{aligned}
$$

Namely, if $a / 2-1 / 4 a<0, a / 2-1 / 4 a-1<0$, and $a-1 / 2 a-$ $b<0$, which implies that the conclusion is true. Similarly, for the controllers (23), we can still use the method to obtain the estimation.
Remark 8. (1) The nonlinear feedback controllers can be used to simultaneously obtain (i) hybrid lag synchronization for $\tau>0$, (ii) hybrid anticipated synchronization for $\tau<0$, and (iii) hybrid complete synchronization for $\tau=0$ between the master system (19) and the slave system (20).

(2) Although the above-obtained feedback controllers are nonlinear, they are simpler than those of the so-called natural control controllers, which are derived by using with a simple stable matrix $M$ and $N$ for the master system (19) and the slave system (20). 


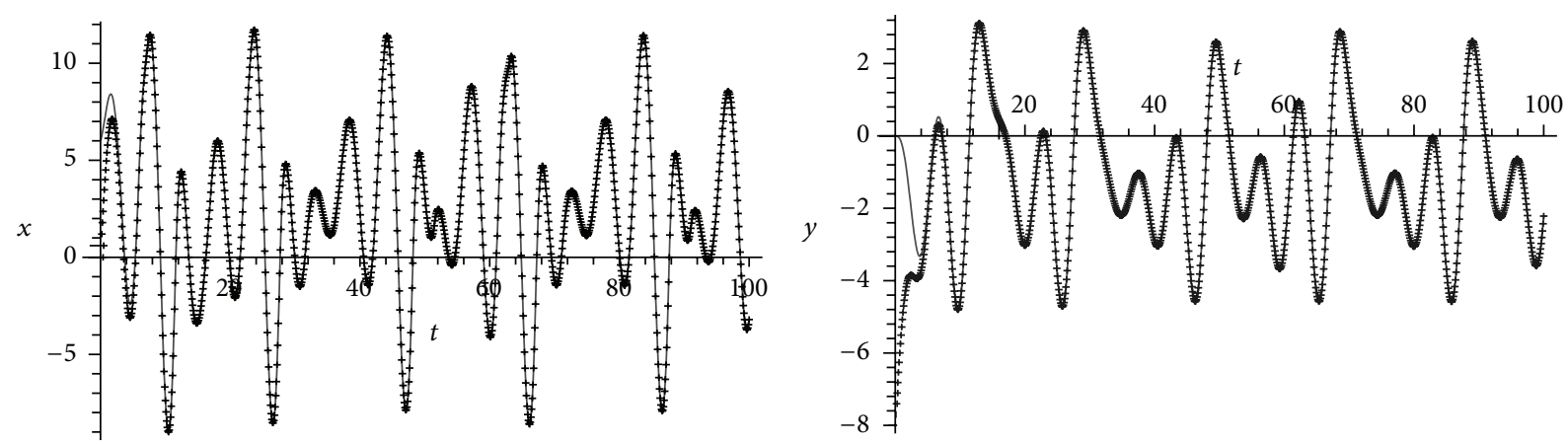

(a)

(b)

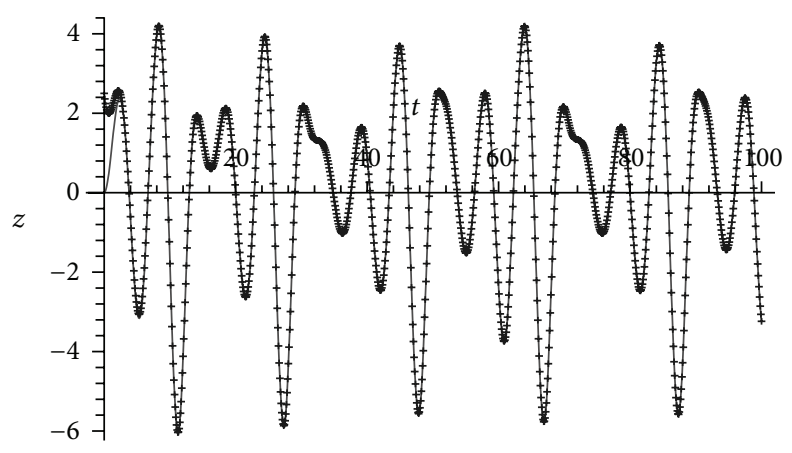

(c)

Figure 6: The solutions of the master and slave systems with control law. (a) Signals $x_{m}$ (the dashed line) and $x_{s}$ (the solid line). (b) Signals $y_{m}$ (the dashed line) and $y_{s}$ (the solid line). (c) Signals $z_{m}$ (the dashed line) and $z_{s}$ (the solid line).

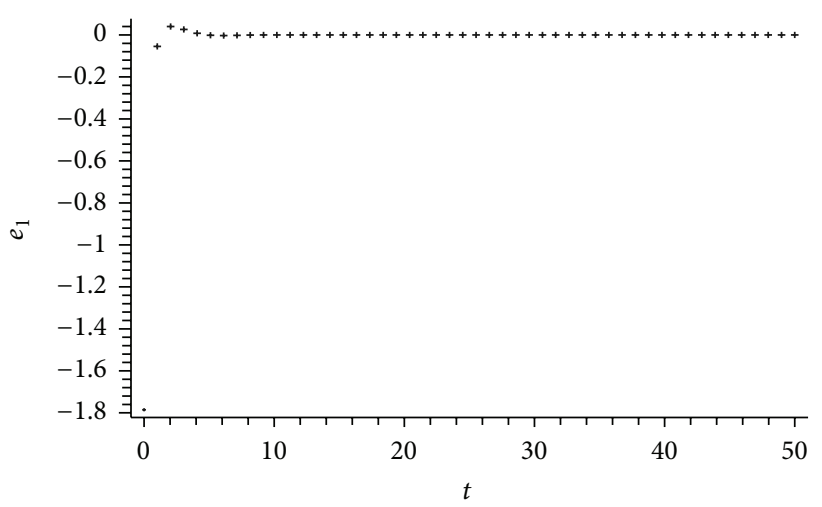

(a)

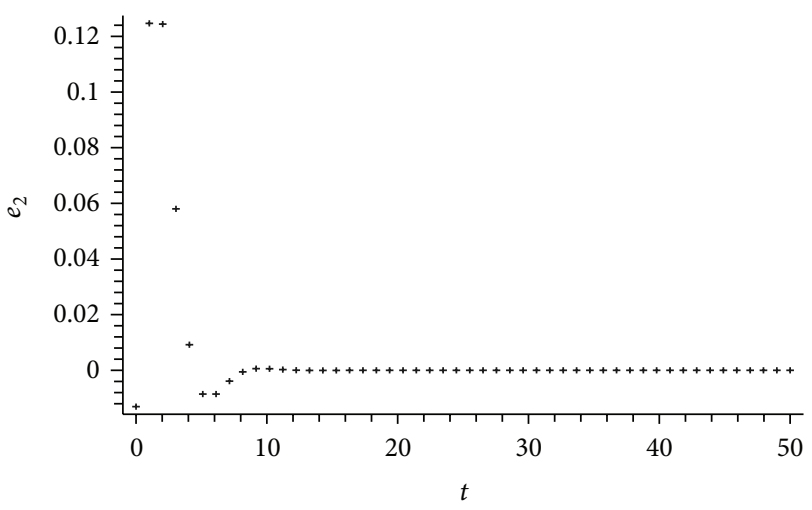

(b)

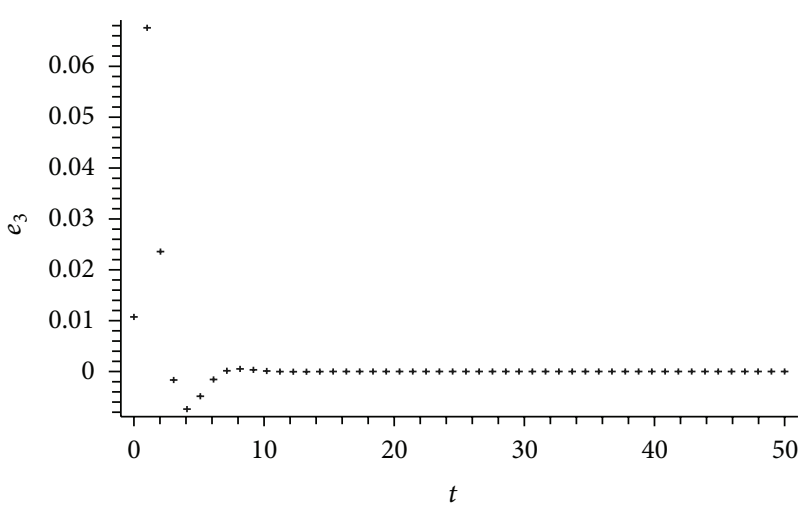

(c)

FIGURE 7: The orbits of error states: (a) $e_{1}=\delta_{1} x_{m}(t)-\epsilon_{1} x_{s}(t-\tau)$, (b) $e_{2}=\delta_{2} y_{m}(t)-\epsilon_{2} y_{s}(t-\tau)$, and (c) $e_{3}=\delta_{3} z_{m}(t)-\epsilon_{3} z_{s}(t-\tau)$. 


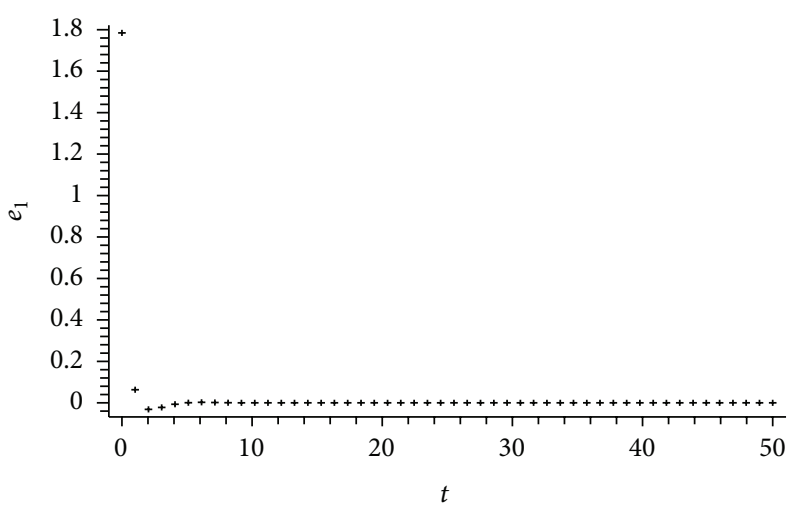

(a)

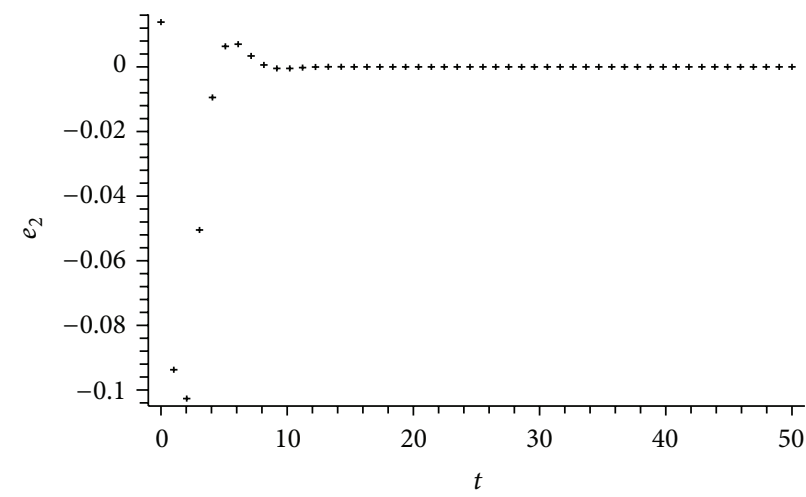

(b)

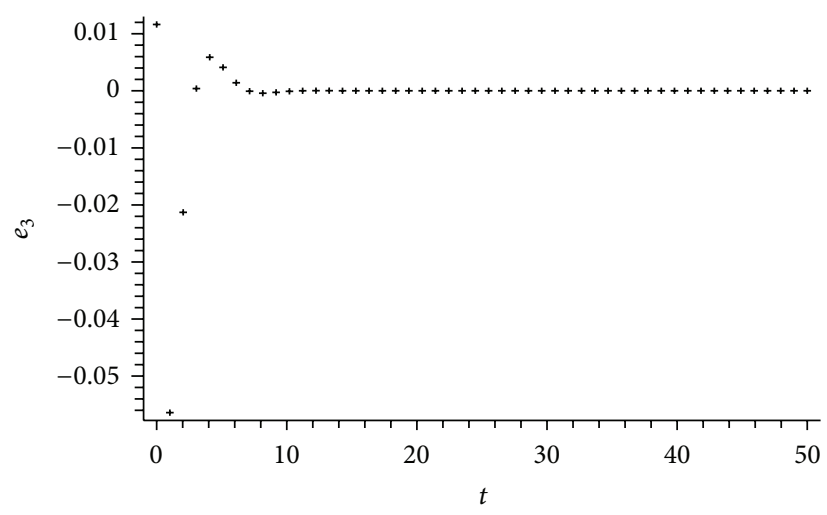

(c)

FIgURE 8: The orbits of error states: (a) $e_{1}=\delta_{1} x_{m}(t)-\epsilon_{1} x_{s}(t-\tau)$, (b) $e_{2}=\delta_{2} y_{m}(t)-\epsilon_{2} y_{s}(t-\tau)$, and (c) $e_{3}=\delta_{3} z_{m}(t)-\epsilon_{3} z_{s}(t-\tau)$.

In the following, we obtain the numerical simulations results to prove the effective control. Numerical simulations results are presented to demonstrate the effectiveness of the proposed synchronization methods. The parameters are chosen to be $[a, b, c, d]=[-2,0.4,1.62,3]$ in all simulations so that the chaotic system exhibits a chaotic behavior if no control is applied. The initial value $\left[x_{m}(0), y_{m}(0), z_{m}(0)\right]$ is taken as the random number $[0,0,0]$ and $\left[x_{s}(0), y_{s}(0)\right.$, $\left.z_{s}(0)\right]=[3,4,5]$. The parameters $\left[\delta_{1}, \delta_{2}, \delta_{3}, \epsilon_{1}, \epsilon_{2}, \epsilon_{3}\right]=[2$, $-2,0.5,2,1,3]$.

Case 1. Hybrid complete projective control: in the case $\tau=$ 0 , without loss of generality, the initial values of the error dynamical system $(21)$ are $e_{1}(0)=\delta_{1} x_{m}(0)-\epsilon_{1} x_{s}(0)=-6$, $e_{2}(0)=\delta_{2} y_{m}(0)-\epsilon_{2} y_{s}(0)=8$, and $e_{3}(0)=\delta_{3} z_{m}(0)-\epsilon_{3} z_{s}(0)=$ -2.5 . The dynamics of hybrid complete control errors for the master system (19) and the slave system (20) is displayed in Figures 4, 5, and 6. Figure 4 shows the chaotic attractors of the master and slave systems with different initial values in the same coordinate. Figures 5(a)-5(c) show the evolutions of the error functions $e_{1}, e_{2}$, and $e_{3}$. Figures $6(\mathrm{a})-6(\mathrm{c})$ the solutions of the master and slave systems with control law.

Case 2. Hybrid lag projective control: in the case $\tau>0$, without loss of generality, we set $\tau=0.3$. Thus the initial values of the error dynamical system (21) are $e_{1}(0)=\delta_{1} x_{m}(0)-$ $\epsilon_{1} x_{s}(-0.3)=1.784475527, e_{2}(0)=\delta_{2} y_{m}(0)-\epsilon_{2} y_{s}(-0.3)=$ 0.01384929027 , and $e_{3}(0)=\delta_{3} z_{m}(0)-\epsilon_{3} z_{s}(-0.3)=$ 0.01162129660 . For simplification, we only give the dynamics of the evolutions of hybrid lag control errors for the master system (19) and the slave system (20) displayed in Figure 7.

Case 3. Hybrid anticipated projective control: in the case $\tau<0$, without loss of generality, we set $\tau=-0.3$. Thus the initial values of the error dynamical system (21) are $e_{1}(0)=$ $\delta_{1} x_{m}(0)-\epsilon_{1} x_{s}(-0.3)=-1.785359335, e_{2}(0)=\delta_{2} y_{m}(0)-$ $\epsilon_{2} y_{s}(-0.3)=-0.01304389936$, and $e_{3}(0)=\delta_{3} z_{m}(0)-$ $\epsilon_{3} z_{s}(-0.3)=0.01072905365$. For simplification, we only give the dynamics of the evolutions of hybrid lag control errors for the master system (19) and the slave system (20) as displayed in Figure 8.

\section{Applications of the LACHPS Control Method via Cascade Control Idea}

In the section, based on the idea of cascade approach $[42,50$, 51], we achieve the effectiveness control idea.

Firstly, we take the system (18) as master system. The slave system is given by

$$
\begin{gathered}
\dot{X}(t-\tau)=a z(t-\tau)-c|Q(t-\tau)|+d+u_{1}, \\
\dot{Q}(t-\tau)=a z(t-\tau)+u_{2},
\end{gathered}
$$




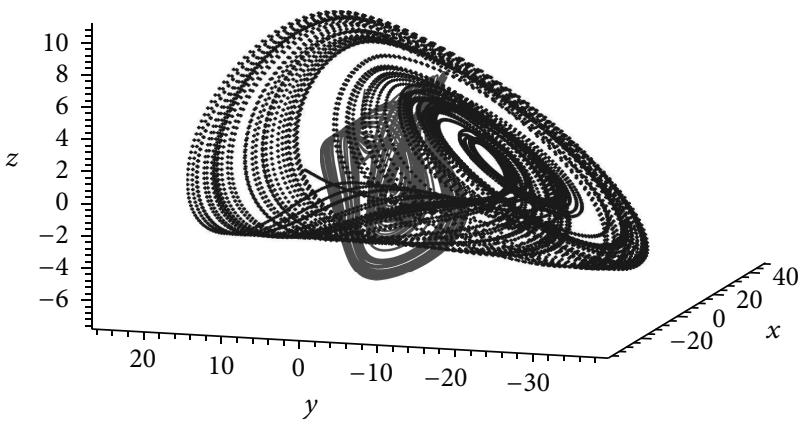

(a)

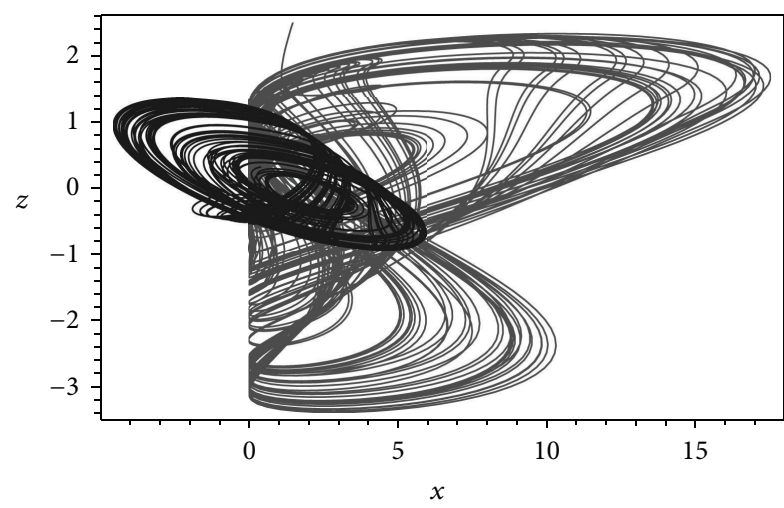

(c)

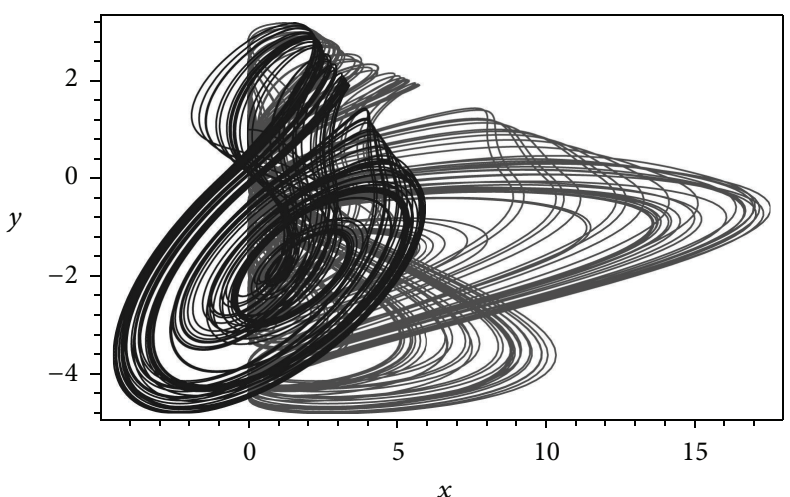

(b)

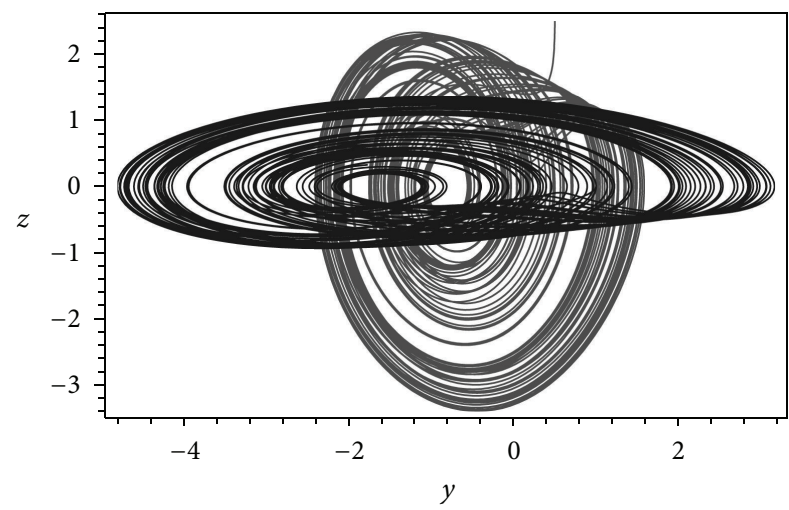

(d)

Figure 9: (a) The synchronized attractors in $(x, y, z)$ space and the phase figure in the different space (b) $(x, y)$ space, (c) $(x, z)$ space, (d) $(y, z)$ space " - -" denotes for the master system, “..." denotes for the slave system synchronized.

where $\left(u_{1}, u_{2}\right)^{T}$ is external control functions that is to be designed below. follows:

Let the error states functions of systems (18) and (29) as

$$
e_{1}=X(t-\tau)-\epsilon_{1} x(t), \quad e_{2}=Q(t-\tau)-\epsilon_{2} y(t),
$$

where $\epsilon_{1}=a_{11} x(t)+a_{12}, \epsilon_{2}=a_{21} y(t)+a_{22}$, and $\tau$ is the time lag or anticipated. The goal of the control is to find a controller $\left(u_{1}, u_{2}\right)^{T}$ such that the states of the master system (18) and the states of the slave system (29) are globally synchronized asymptotically; that is,

$$
\begin{aligned}
& \lim _{t \rightarrow \infty}\left\|X(t-\tau)-\epsilon_{1} x(t)\right\|=0, \\
& \lim _{t \rightarrow \infty}\left\|Q(t-\tau)-\epsilon_{2} y(t)\right\|=0 .
\end{aligned}
$$

Let us define the Lyapunov functions as

$$
V_{1}=\frac{1}{2}\left(e_{1}^{2}+e_{2}^{2}\right)
$$

If the Lyapunov function (32) satisfies the conditions

$$
\begin{array}{ll}
V_{1}>0 & \text { if }\left(e_{1}, e_{2}\right) \neq(0,0), \\
V_{1}=0 & \text { if }\left(e_{1}, e_{2}\right)=(0,0),
\end{array}
$$

$$
\begin{array}{ll}
\dot{V}_{1}<0 & \text { if }\left(e_{1}, e_{2}\right) \neq(0,0), \\
\dot{V}_{1}=0 & \text { if }\left(e_{1}, e_{2}\right)=(0,0) .
\end{array}
$$

then $e_{i}(i=1,2)$ will asymptotically tend to zero and

$$
\begin{aligned}
& \lim _{t \rightarrow \infty}\left\|X(t-\tau)-\epsilon_{1} x(t)\right\|=0, \\
& \lim _{t \rightarrow \infty}\left\|Q(t-\tau)-\epsilon_{2} y(t)\right\|=0 .
\end{aligned}
$$

With the aid of Maple and we omit the details by the aid of Maple soft, we choose

$$
\begin{aligned}
u_{1}= & -e_{1}(t)-a z(t-\tau)+c|Q(t-\tau)|-d \\
& +2 a_{11} x(t) a z(t)-2 a_{11} x(t) c|y(t)|+2 a_{11} x(t) d \\
& +a_{12} a z(t)-a_{12} c|y(t)|+a_{12} d+k_{1}, \\
u_{2}= & -e_{2}(t)-a z(t-\tau) \\
& +2 a_{21} a z(t) y(t)+a z(t) a_{22}+k_{2}
\end{aligned}
$$

then (18) and (29) will be satisfied. Next we take (29) as the master system, and the slave one is as follows:

$$
\dot{Y}(t-\tau)=a Z(t-\tau)+u_{3},
$$




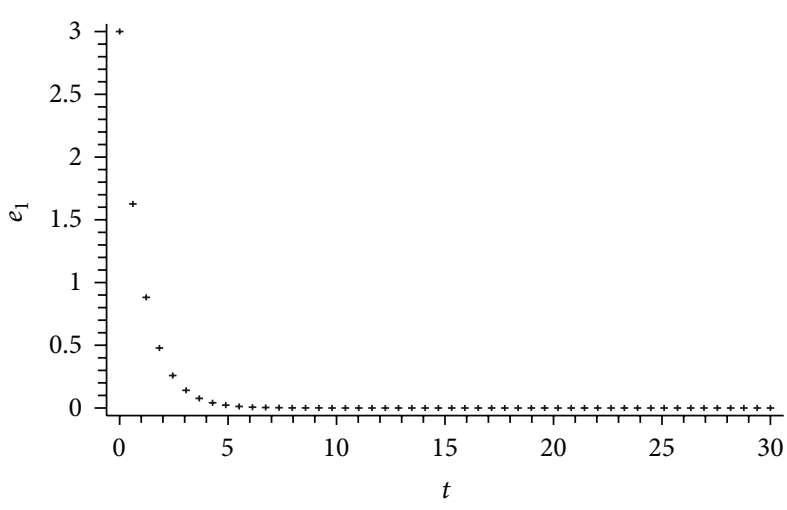

(a)

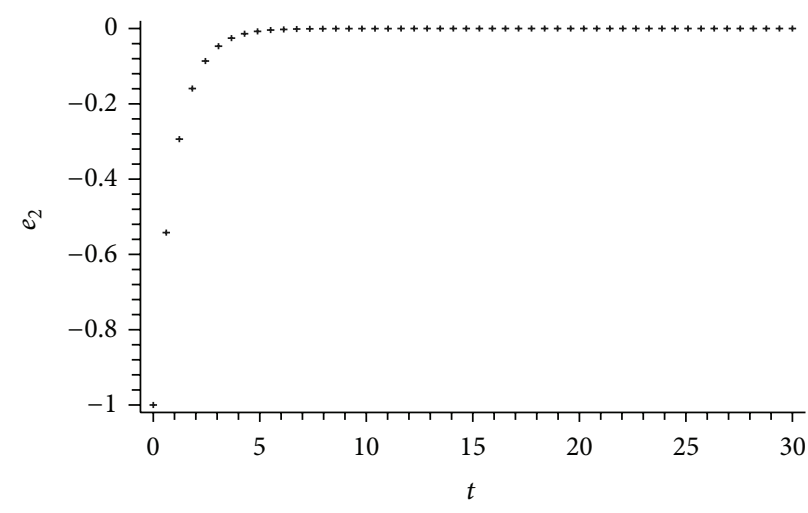

(b)

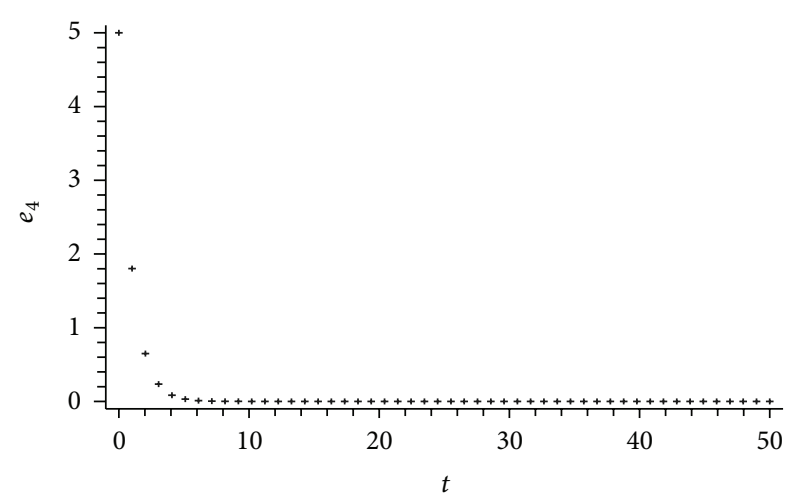

(c)

FIgURE 10: The orbits of error states: (a) $e_{1}=X(t-\tau)-\epsilon_{1} x(t)$, (b) $e_{3}=Y(t-\tau)-\epsilon_{3} y(t)$, and (c) $e_{4}=Z(t-\tau)-\epsilon_{4} z(t)$.

$$
\dot{Z}(t-\tau)=-\frac{x(t-\tau)}{2 a}-\frac{y(t-\tau)}{2 a}-b z(t-\tau)+u_{4},
$$

where $\left(u_{3}, u_{4}\right)^{T}$ is a desired controller.

The relevant Lyapunov function can be chosen as

$$
V_{2}=\frac{1}{2}\left(e_{3}^{2}+e_{4}^{2}\right)
$$

where $e_{3}=Y(t-\tau)-\epsilon_{3} y(t), e_{4}=Z(t-\tau)-\epsilon_{4} z(t), \epsilon_{3}=a_{31} y(t)+$ $a_{32}, \epsilon_{4}=a_{41} z(t)+a_{42}$, and $\tau$ is the time lag or anticipated. We take $u_{3}, u_{4}$ as

$$
\begin{aligned}
& u_{3}=-Y(t-\tau)+a_{31} y(t)^{2}+y(t) a_{32}-a Z(t-\tau) \\
& +2 a_{31} a z(t) y(t)+a z(t) a_{32}+k_{3} \\
& u_{4}=-\frac{1}{2 a}\left[2 a Z(t-\tau)-2 a a_{41} z(t)^{2}\right. \\
& -2 a a_{42} z(t)-Y(t-\tau)+a_{42} x(t) \\
& +a_{42} y(t)+2 a_{42} b z(t) a+2 a_{41} z(t) y(t) \\
& -X(t-\tau)-2 b Z(t-\tau) a \\
& \left.+2 a_{41} z(t) x(t)+4 a_{41} z(t)^{2} b a-2 k_{4} a\right]
\end{aligned}
$$

which make $e_{3}$ and $e_{4}$ approach to zero when $t \rightarrow+\infty$. Therefor the LACHPS is achieved for the systems (29) and (36) via cascade method.

For simplicity and illustration, the parameters $k_{1}=2$, $k_{2}=3, k_{3}=4, k_{4}=2$, we consider $\left[a_{11}, a_{12}, a_{21}, a_{21}, a_{31}, a_{32}\right.$, $\left.a_{41}, a_{42}\right]=[1,0,0,3,0,2,0,-5]$ and the initial values $[x(0)$, $y(0), z(0), X(0), Y(0), Z(0), Q(0)]=[0,1,1,3,1,5,-5]$. We may only choose $\tau=0$; other cases are similar. Figure 9 shows the LACHPS via cascade method for the system (18). And Figures 10(a)-10(c) show the numeric simulations of the error functions $e_{1}, e_{3}$, and $e_{4}$.

\section{Conclusion}

In this paper, based on the stability theory and an active control technique, we investigate the lag, anticipated, and complete hybrid projective synchronization control (LACHPS) for nonlinear chaotic systems. A nonlinear controller has been proposed to achieve lag, anticipated, and complete projective synchronization of chaotic systems. The proposed synchronization is simple and theoretically rigorous. Colpitts oscillators are used to illustrate the effectiveness of the proposed synchronization scheme. It should be note that lag synchronization control, anticipated synchronization control, and complete synchronization control. Therefore, the results of this paper are more applicable and representative. 


\section{Appendix}

Proof of Proposition 3. According to $e=M x(t)-N y(t-\tau)$ in definition of LACHPS, one can get

$$
\begin{aligned}
& \dot{e}(t)= M \dot{x}(t)-\dot{N} y(t-\tau)-N \dot{y}(t-\tau) \\
&= M[\phi x(t)+F(x(t), t)]-h y(t-\tau) \\
&-N(\phi y(t-\tau)+G(y(t-\tau), t)+U) \\
&= \phi(M x(t)-N y(t-\tau))+M F(x(t), t) \\
&-h y(t-\tau)-N G(y(t-\tau), t) \\
&-N\left[-N^{-1} h y(t-\tau)\right. \\
& \quad+N^{-1} M F(x(t), t)-G(y(t-\tau), t) \\
&\left.\quad-N^{-1} K(M x(t)-N y(t-\tau))\right] \\
&=(\phi+K) e(t) .
\end{aligned}
$$

We solve the above equation $\dot{e}(t)=(\phi+K) e(t)$, and

$$
\|e(t)\|=\left\|e^{(\phi+K) t} e(0)\right\| \text {. }
$$

Because the real parts of all the eigenvalues of $(\phi+K)$ are negative, $\|e(t)\| \rightarrow 0$ if $t \rightarrow 0$. Namely, $\lim _{t \rightarrow 0}\|e(t)\|=0$. For a feasible control, the feedback $K$ must be selected such that all the eigenvalues of $(\phi+K)$, if any, have negative real parts. Thus, if the matrix $(\phi+K)$ is in full rank, the system $\dot{e}$ is asymptotically stable at the origin, which implies that (1) and (2) are in the state of LACHPS control.

Proof of Proposition 4. From $e=M x(t)-N y(t-\tau)$ in definition of LACHPS, one can get

$$
\begin{aligned}
\dot{e}(t)= & \dot{M} x(t)+M \dot{x}(t)-N \dot{y}(t-\tau) \\
= & M[\phi x(t)+F(x(t), t)]+g x(t) \\
& -N(\phi y(t-\tau)+G(y(t-\tau), t)+U) \\
= & \phi(M x(t)-N y(t-\tau)) \\
& +M F(x(t), t)+g x(t)-N G(y(t-\tau), t) \\
& -N\left[-N^{-1} g x(t)+N^{-1} M F(x(t), t)\right. \\
& \quad-G(y(t-\tau), t) \\
& \left.-N^{-1} K[M x(t)-N y(t-\tau)]\right] \\
= & (\phi+K) e(t) .
\end{aligned}
$$

We solve the above equation $\dot{e}(t)=(\phi+K) e(t)$, and

$$
\|e(t)\|=\left\|e^{(\phi+K) t} e(0)\right\| .
$$

Because the real parts of all the eigenvalues of $(\phi+K)$ are negative, $\|e(t)\| \rightarrow 0$ if $t \rightarrow 0$. Namely, $\lim _{t \rightarrow 0}\|e(t)\|=0$.
For a feasible control, the feedback $K$ must be selected such that all the eigenvalues of $(\phi+K)$, if any, have negative real parts. Thus, if the matrix $(\phi+K)$ is in full rank, the system $\dot{e}$ is asymptotically stable at the origin, which implies that (1) and (2) are in the state of LACHPS control.

In this case, the active control method [22] is usually adopted to obtain the gain matrix $K$ for any specified eigenvalues of $(\phi+K)$.

\section{Conflict of Interests}

The authors declare that there is no conflict of interests regarding the publication of this paper.

\section{Acknowledgments}

The authors are deeply indebted to Professor Yulin zhao of Sun Yat-sen University and Professor Zheng-an Yao of Sun Yat-sen University for giving some help and suggestion. This work is supported by the NSF of China (no. 11171355), the Ph.D. Programs Foundation of Ministry of Education of China (no. 20100171110040), Guangdong Provincial culture of seedling of China (no. 2013LYM0081), and Guangdong Provincial NSF of China (no. S2012010010069), the Shaoguan Science and Technology Foundation (no. 313140546), and Science Foundation of Shaoguan University. The authors thank the handling editor and the reviewers for their valuable comments and suggestions, which improved the completeness of the paper.

\section{References}

[1] J. Lü and G. Chen, "A new chaotic attractor coined," International Journal of Bifurcation and Chaos in Applied Sciences and Engineering, vol. 12, no. 3, pp. 659-661, 2002.

[2] J. Lü, G. Chen, D. Cheng, and S. Celikovsky, "Bridge the gap between the Lorenz system and the Chen system," International Journal of Bifurcation and Chaos in Applied Sciences and Engineering, vol. 12, no. 12, pp. 2917-2926, 2002.

[3] T. Zhou, G. Chen, and Q. Yang, "Constructing a new chaotic system based on the Silnikov criterion," Chaos, Solitons and Fractals, vol. 19, no. 4, pp. 985-993, 2004.

[4] T. Zhou, Y. Tang, and G. Chen, "Chen's attractor exists," International Journal of Bifurcation and Chaos in Applied Sciences and Engineering, vol. 14, no. 9, pp. 3167-3177, 2004.

[5] T. Zhou, Y. Tang, and G. Chen, "Complex dynamical behaviors of the chaotic Chen's system," International Journal of Bifurcation and Chaos in Applied Sciences and Engineering, vol. 13, no. 9, pp. 2561-2574, 2003.

[6] T. Zhou and G. Chen, "Classification of chaos in 3-D autonomous quadratic systems. I. Basic framework and methods," International Journal of Bifurcation and Chaos in Applied Sciences and Engineering, vol. 16, no. 9, pp. 2459-2479, 2006.

[7] G. Qi, G. Chen, S. Du, Z. Chen, and Z. Yuan, "Analysis of a new chaotic system," Physica A, vol. 352, no. 2-4, pp. 295-308, 2005.

[8] Q. Yang, G. Chen, and T. Zhou, "A unified Lorenz-type system and its canonical form," International Journal of Bifurcation and Chaos in Applied Sciences and Engineering, vol. 16, no. 10, pp. 2855-2871, 2006. 
[9] Q. Yang, G. Chen, and K. Huang, "Chaotic attractors of the conjugate Lorenz-type system," International Journal of Bifurcation and Chaos in Applied Sciences and Engineering, vol. 17, no. 11, pp. 3929-3949, 2007.

[10] Q. Yang and G. Chen, "A chaotic system with one saddle and two stable node-foci," International Journal of Bifurcation and Chaos in Applied Sciences and Engineering, vol. 18, no. 5, pp. 1393-1414, 2008.

[11] E. M. Elabbasy, H. N. Agiza, and M. M. El-Dessoky, "Adaptive synchronization for four-scroll attractor with fully unknown parameters," Physics Letters A, vol. 349, no. 1-4, pp. 187-191, 2006.

[12] T. M. Hoang and M. Nakagawa, "Projective-lag synchronization of coupled multidelay feedback systems," Journal of the Physical Society of Japan, vol. 75, no. 9, Article ID 094801, 2006.

[13] Q. Miao, Y. Tang, S. Lu, and J. Fang, "Lag synchronization of a class of chaotic systems with unknown parameters," Nonlinear Dynamics, vol. 57, no. 1-2, pp. 107-112, 2009.

[14] C. Li, X. Liao, and K.-w. Wong, "Chaotic lag synchronization of coupled time-delayed systems and its applications in secure communication," Physica D, vol. 194, no. 3-4, pp. 187-202, 2004.

[15] D. Wang, Y. Zhong, and S. Chen, "Lag synchronizing chaotic system based on a single controller," Communications in Nonlinear Science and Numerical Simulation, vol. 13, no. 3, pp. 637644, 2008.

[16] G. M. Mahmoud and T. Bountis, "The dynamics of systems of complex nonlinear oscillators: a review," International Journal of Bifurcation and Chaos in Applied Sciences and Engineering, vol. 14, no. 11, pp. 3821-3846, 2004.

[17] Z. Yan, "Q-S synchronization in 3D Henon-like map and generalized Henon map via a scalar controller," Physics Letters A, vol. 342, pp. 309-317, 2005.

[18] Z. Yan, "Q-S (lag or anticipated) synchronization backstepping scheme in a class of continuous-time hyperchaotic systems-A symbolic-numeric computation approach," Chaos, vol. 15, no. 2, Article ID 023902, 2005.

[19] Z. Yan, "Controlling hyperchaos in the new hyperchaotic Chen system," Applied Mathematics and Computation, vol. 168, no. 2, pp. 1239-1250, 2005.

[20] Z. Yan and P. Yu, "Hyperchaos synchronization and control on a new hyperchaotic attractor," Chaos, Solitons and Fractals, vol. 35, no. 2, pp. 333-345, 2008.

[21] M. Hu, Z. Xu, R. Zhang, and A. Hu, "Adaptive full state hybrid projective synchronization of chaotic systems with the same and different order," Physics Letters A, vol. 365, no. 4, pp. 315-327, 2007.

[22] L. M. Pecora and T. L. Carroll, "Synchronization in chaotic systems," Physical Review Letters, vol. 64, no. 8, pp. 821-824, 1990.

[23] M. M. Al-sawalha and M. S. M. Noorani, "Anti-synchronization of chaotic systems with uncertain parameters via adaptive control," Physics Letters A, vol. 373, no. 32, pp. 2852-2857, 2009.

[24] G.-H. Li, "Generalized projective synchronization between Lorenz system and Chen's system," Chaos, Solitons and Fractals, vol. 32, no. 4, pp. 1454-1458, 2007.

[25] G.-H. Li, "Modified projective synchronization of chaotic system," Chaos, Solitons and Fractals, vol. 32, no. 5, pp. 1786-1790, 2007.

[26] C. Chicone, Ordinary Differential Equations with Applications, vol. 34 of Texts in Applied Mathematics, Springer, New York, NY, USA, 2nd edition, 2006.
[27] M. Fečkan, Topological Degree Approach to Bifurcation Problems, vol. 5 of Topological Fixed Point Theory and Its Applications, Springer, New York, NY, USA, 2008.

[28] Z.-L. Wang, "Projective synchronization of hyperchaotic Lü system and Liu system," Nonlinear Dynamics, vol. 59, no. 3, pp. 455-462, 2010.

[29] G.-H. Li, "Projective lag synchronization in chaotic systems," Chaos, Solitons and Fractals, vol. 41, no. 5, pp. 2630-2634, 2009.

[30] A. C. J. Luo, "A theory for synchronization of dynamical systems," Communications in Nonlinear Science and Numerical Simulation, vol. 14, no. 5, pp. 1901-1951, 2009.

[31] G. M. Mahmoud, S. A. Aly, and A. A. Farghaly, "On chaos synchronization of a complex two coupled dynamos system," Chaos, Solitons and Fractals, vol. 33, no. 1, pp. 178-187, 2007.

[32] G. M. Mahmoud, M. A. Al-Kashif, and S. A. Aly, "Basic properties and chaotic synchronization of complex Lorenz system," International Journal of Modern Physics C, vol. 18, no. 2, pp. 253-265, 2007.

[33] G. M. Mahmoud, T. Bountis, and E. E. Mahmoud, "Active control and global synchronization of the complex Chen and Lü systems," International Journal of Bifurcation and Chaos in Applied Sciences and Engineering, vol. 17, no. 12, pp. 4295-4308, 2007.

[34] G. M. Mahmoud, E. E. Mahmoud, and M. E. Ahmed, "A hyperchaotic complex Chen system and its dynamics," International Journal of Applied Mathematics \& Statistics, vol. 12, no. D07, pp. 90-100, 2007.

[35] G. M. Mahmoud, M. E. Ahmed, and E. E. Mahmoud, "Analysis of hyperchaotic complex Lorenz systems," International Journal of Modern Physics C, vol. 19, no. 10, pp. 1477-1494, 2008.

[36] G. M. Mahmoud, M. A. Al-Kashif, and A. A. Farghaly, "Chaotic and hyperchaotic attractors of a complex nonlinear system," Journal of Physics A, vol. 41, no. 5, Article ID 055104, 2008.

[37] G. M. Mahmoud, S. A. Aly, and M. A. AL-Kashif, "Dynamical properties and chaos synchronization of a new chaotic complex nonlinear system," Nonlinear Dynamics, vol. 51, no. 1-2, pp. 171$181,2008$.

[38] G. M. Mahmoud, T. Bountis, G. M. AbdEl-Latif, and E. E. Mahmoud, "Chaos synchronization of two different chaotic complex Chen and Lü systems," Nonlinear Dynamics, vol. 55, no. 1-2, pp. 43-53, 2009.

[39] M. Han, "On Hopf cyclicity of planar systems," Journal of Mathematical Analysis and Applications, vol. 245, no. 2, pp. 404422, 2000.

[40] M. Han, "The Hopf cyclicity of Lienard systems," Applied Mathematics Letters, vol. 14, no. 2, pp. 183-188, 2001.

[41] M. Han, G. Chen, and C. Sun, "On the number of limit cycles in near-Hamiltonian polynomial systems," International Journal of Bifurcation and Chaos in Applied Sciences and Engineering, vol. 17, no. 6, pp. 2033-2047, 2007.

[42] C. Li and J. Yan, "Generalized projective synchronization of chaos: the cascade synchronization approach," Chaos, Solitons \& Fractals, vol. 30, no. 1, pp. 140-146, 2006.

[43] Y. Chen and X. Li, "Function projective synchronization between two identical chaotic systems," International Journal of Modern Physics C, vol. 18, no. 5, pp. 883-888, 2007.

[44] X. Li and Y. Chen, "Function projective synchronization of two identical new hyperchaotic systems," Communications in Theoretical Physics, vol. 48, no. 5, pp. 864-873, 2007.

[45] G. M. Mahmoud and E. E. Mahmoud, "Modified projective lag synchronization of two nonidentical hyperchaotic complex 
nonlinear systems," International Journal of Bifurcation and Chaos, vol. 21, no. 8, pp. 2369-2379, 2011.

[46] Y. Li, Y. Chen, and B. Li, "Adaptive control and function projective synchronization in $2 \mathrm{~d}$ discrete-time chaotic systems," Communications in Theoretical Physics, vol. 51, no. 2, pp. 270278, 2009.

[47] Y. Li, B. Li, and Y. Chen, "Adaptive function projective synchronization of discrete-time chaotic systems," Chinese Physics Letters, vol. 26, no. 4, Article ID 040504, 2009.

[48] Y. Li, B. Li, and Y. Chen, "Anticipated function synchronization with unknown parameters of discrete-time chaotic systems," International Journal of Modern Physics C, vol. 20, no. 4, pp. 597608, 2009.

[49] Y. Li and B. Li, "Chaos control and projective synchronization of a chaotic Chen-Lee system," Chinese Journal of Physics, vol. 47, no. 3, pp. 261-270, 2009.

[50] H. B. Fotsin and J. Daafouz, "Adaptive synchronization of uncertain chaotic colpitts oscillators based on parameter identification," Physics Letters A, vol. 339, no. 3-5, pp. 304-315, 2005.

[51] Y. Li and C. L. Zheng, "The complex network synchronization via chaos control nodes," Journal of Applied Mathematics, vol. 2013, Article ID 823863, 11 pages, 2013. 


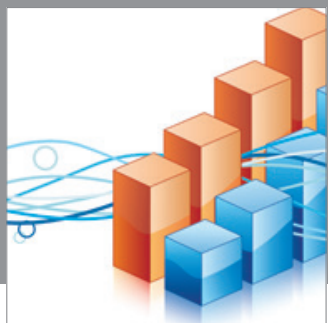

Advances in

Operations Research

mansans

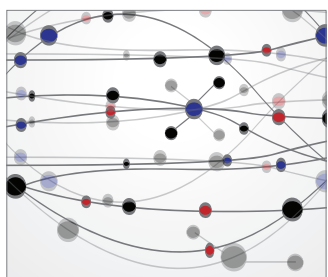

The Scientific World Journal
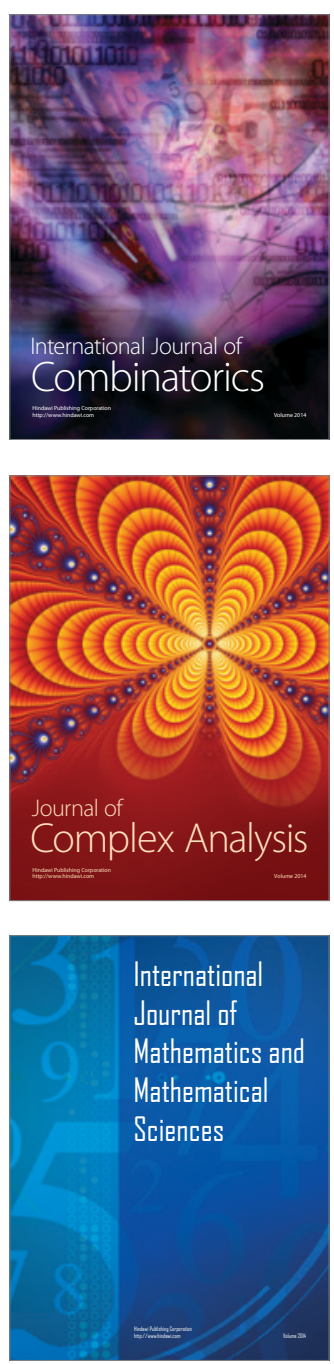
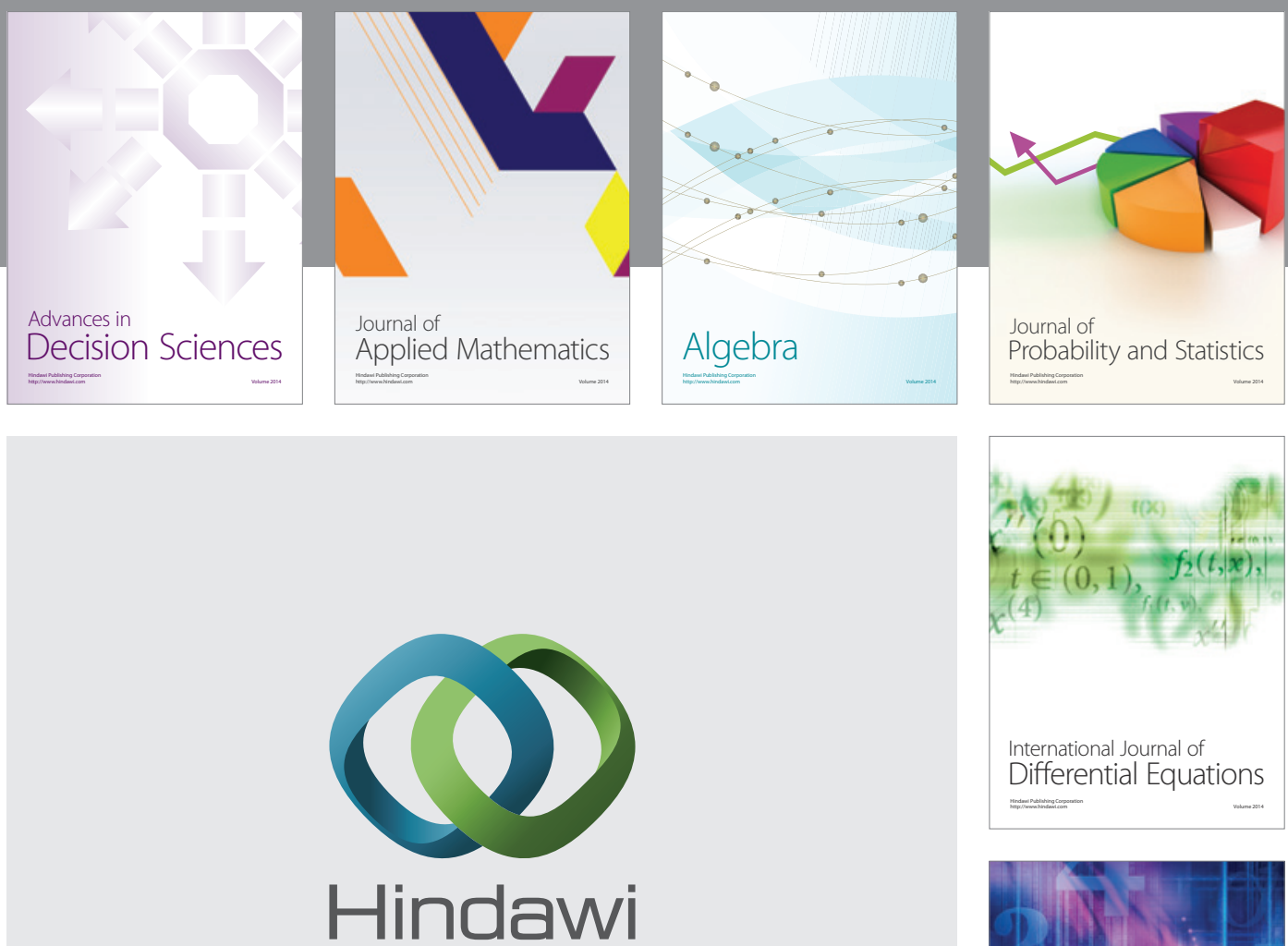

Submit your manuscripts at http://www.hindawi.com
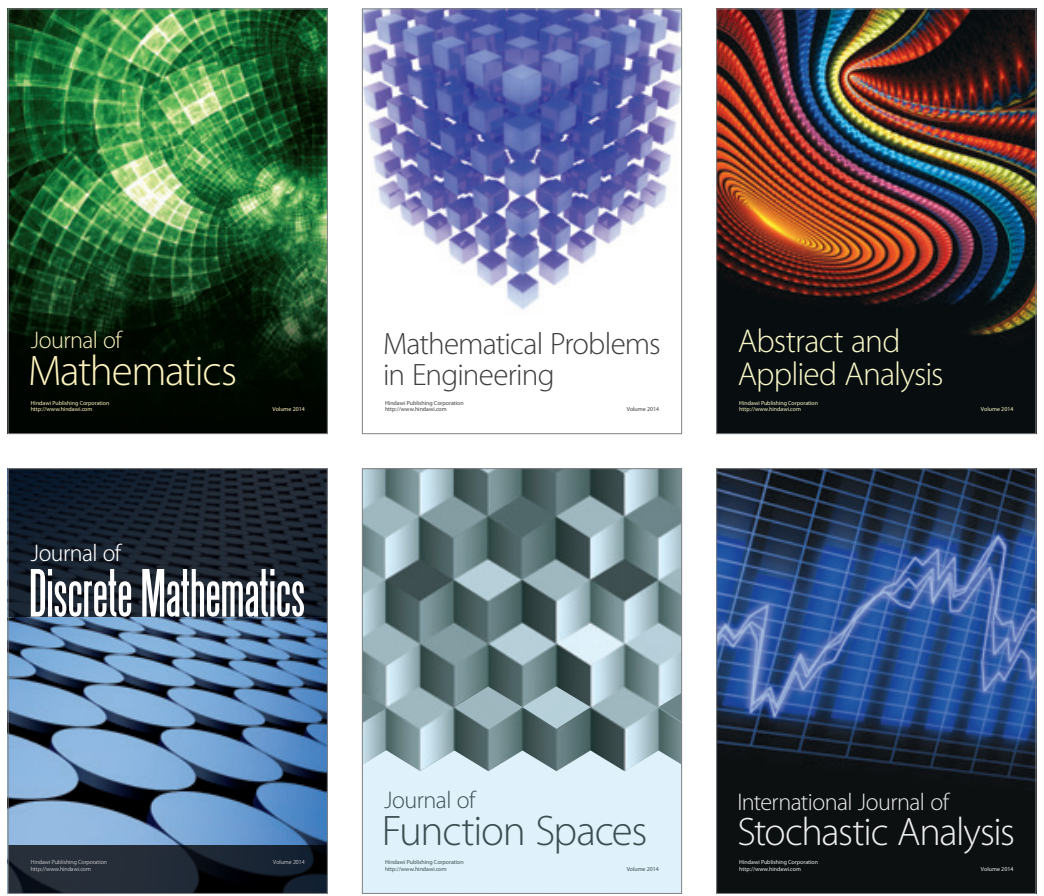

Journal of

Function Spaces

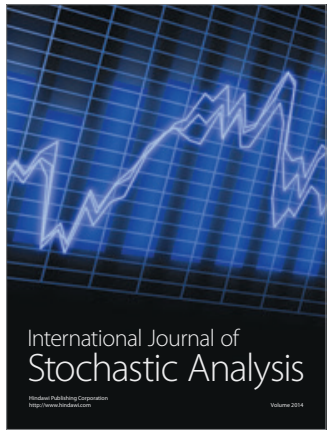

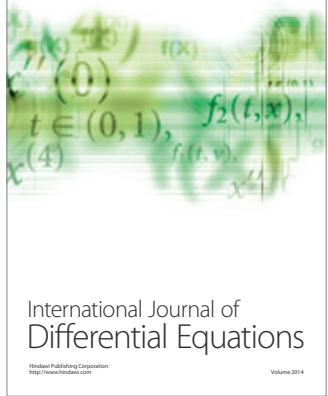
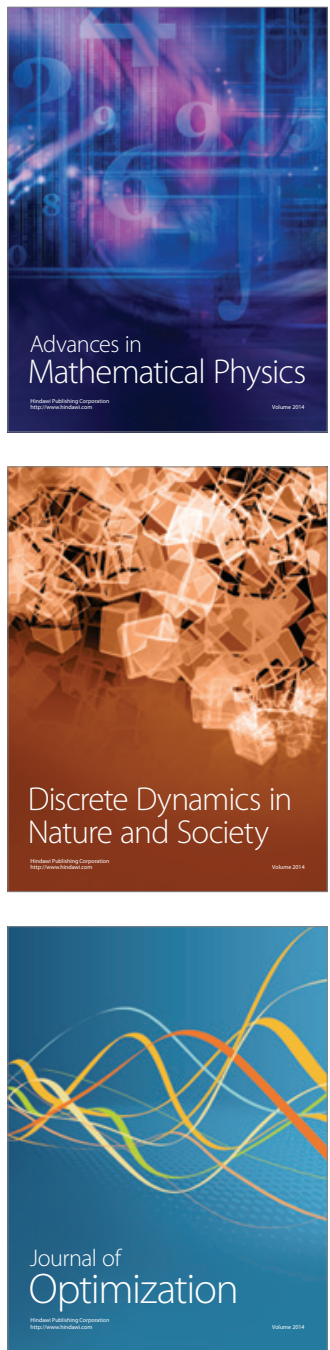